\title{
Formulation and Implementation of a High-Order 3-D Domain Integral Method for the Extraction of Energy Release Rates
}

\author{
H. Ozer, C.A. Duarte*and I.L. Al-Qadi \\ Department of Civil and Environmental Engr., University of Illinois at Urbana-Champaign, \\ Newmark Laboratory, 205 North Mathews Avenue, Urbana, Illinois 61801, USA
}

October 13, 2011

\begin{abstract}
This paper presents a three dimensional (3-D) formulation and implementation of a high-order domain integral method for the computation of energy release rate. The method is derived using surface and domain formulations of the $J$-integral and the weighted residual method. The $J$-integral along 3-D crack fronts is approximated by high-order Legendre polynomials. The proposed implementation is tailored for the Generalized/eXtended Finite Element Method and can handle discontinuities arbitrarily located within a finite element mesh. The domain integral calculations are based on the same integration elements used for the computation of the stiffness matrix. Discontinuities of the integrands across crack surfaces and across computational element boundaries are fully accounted for. The proposed method is able to deliver smooth approximations and to capture the boundary layer behavior of the J-integral using tetrahedral meshes. Numerical simulations of mode-I and mixed mode benchmark fracture mechanics examples verify expected convergence rates for the computed energy release rates. The results are also in good agreement with other numerical solutions available in the literature.
\end{abstract}

\section{Introduction}

The evaluation of accurate fracture parameters in complex three dimensional problems remains a significant challenge in the area of computational fracture mechanics. Stress intensity factors (SIF's) and the energy release rate are among key fracture parameters. Contour and domain integral methods have emerged as viable approaches for the determination of these parameters. The $J$-integral is among the most commonly used fracture parameter and was introduced as a path independent integral by Rice [1968] to analyze crack tip strain fields in the context of nonlinear elasticity. The computation of the energy release rate or the $J$ integral using surface or volume domains was initially introduced and employed in the works of Shih et al. [1986], Moran and Shih [1987], and Li et al. [1985]. The domain integral method yields pointwise values of the energy release rate along a three dimensional (3-D) planar or non-planar crack front. It is commonly formulated using a weighted mean of the $J$-integral within each extraction domain along a $3-\mathrm{D}$ crack front. This assumption facilitates the numerical implementation and is acceptable when extraction domains are sufficiently small along the crack front. This approach was successfully implemented in the context of the

${ }^{*}$ Corresponding author. E-mail: caduarte@illinois.edu 
finite element method (FEM) and adopted by many commercial finite element (FE) packages. Although not a mathematical requirement, FE implementations are usually done with the aid of structured meshes of crack front elements. Rings of elements mesh cylindrical domains used in the $J$-integral calculations. There are, however, some examples in the literature of implementations geared towards unstructured FE meshes [Cervenka and Saouma, 1997, Nikishkov and Atluri, 1987].

The use of domain integral approaches within recently emerged numerical methods such as the Generalized or eXtended Finite Element Method (G/XFEM) poses significant challenges to the numerical implementation especially in three dimensional problems. These methods are instances of the partition of unity method [Babuška et al., 1994, Melenk and Babuška, 1996, Duarte and Oden, 1996b,a]. They overcome several mesh design and computational issues of the FEM especially for the problems with discontinuities such as cracks, material interfaces, etc. Early developments of the GFEM and its implementation for solving 3-D elasticity problems can be found in the works of Duarte et al. [2000, 2001]. Recent reviews of Generalized/eXtended FEMs along with a brief history on their development can be found in [Belytschko et al., 2009, Fries and Belytschko, 2010].

Three-dimensional stress intensity factor and energy release rate computations in the XFEM were performed using a domain integral approach as introduced by Sukumar et al. [2000] and Moës et al. [2002]. The implementation of domain integrals in the XFEM, however, differs from the standard FEM on the setup of extraction domains. An alternative approach was followed in these works. A grid of hexahedral integration elements independent from the finite element mesh was used to define the extraction domains and to compute energy release rates. The integrand of the domain integrals contains derivatives of the numerical solution and are thus discontinuous across computational element boundaries. Therefore, accuracy of numerical integration is not guaranteed in this approach.

As an alternative to the domain integral approach commonly used in FE implementations, the Contour Integral Method (CIM) and the Cutoff Function Method (CFM) were adapted for the GFEM [Pereira and Duarte, 2004, 2005, 2006] to compute fracture parameters. These methods are superconvergent techniques for the computation of stress intensity factors and energy release rate. Details on these techniques are presented in the works of Szabo and Babuska [Szabo and Babuška, 1988, 1991]. In three dimensional implementations of these methods, the CFM uses a hollow cylindrical domain to compute SIF's whereas the CIM computes SIF's using a cylindrical surface enclosing the point of interest along the crack front. The CFM and CIM are both based on the computation of some functionals of the numerical solution and the so-called extraction functions. The cutoff function just like the auxiliary $q$-function used in domain integral methods is defined in such a way that the expressions to extract stress intensity factors in the CFM do not contain terms related to the derivatives of the GFEM solution. This is particularly desirable for the implementation of these methods in the GFEM when the extraction domains are independent from the finite element mesh around the crack front. The numerical integration involves functionals containing terms from the asymptotic crack tip expansion and GFEM displacements. However, the CFM and the CIM require the knowledge of the asymptotic expansion of the elasticity solution near the crack front, which may not be available for inelastic materials such as plastic and viscoelastic. A CFM method based on high-order polynomial approximations of edge and vertex stress intensity factors was formulated in [Andersson et al., 1995]. Stress intensity factors and energy release rate are smooth functions along a 3-D crack front for smooth loading and smoothly shaped edges [Andersson et al., 1995]. Thus, polynomial approximations of these functions are very effective.

In this paper we introduce a high-order domain integral method for the extraction of energy release rates along 3-D crack fronts. The method proposed here is based on surface and domain formulations of the J-integral and thus, in contrast with the cut-off function method presented in [Andersson et al., 1995], 
does not require the knowledge of the asymptotic expansion of the elasticity solution in the neighborhood of cracks. The method provides an approximation of the $J$-integral function as a linear combination of Legendre polynomials. As a result, extracted functions are smooth which is important when using them to drive 3-D crack propagation in elastic or inelastic materials. High-order approximations of fracture parameters are also important near the intersections of the crack front with the boundary of the domain since these quantities may exhibit strong gradients at these regions due to changes in the singularity order of the elasticity solution. Fluctuations of numerically extracted fracture mechanics parameters lead in general to wrong crack paths. In the proposed high-order formulation, only a few (three or less in general) extraction domains need to be defined along a 3-D crack front. This is in contrast with the current practice of defining a large number of small extraction domains along a crack front and using a weighted mean of the $J$-integral function within each domain. The potential for extensions of the proposed formulation to inelastic materials such as viscoelastic or plastic is another attractive feature of the proposed approach. To our knowledge, high-order extraction methods are only available for the cut-off function method and have only been implemented in combination with the p-version of the FEM.

We also present an strategy to implement domain integral methods in partition of unity based methods such as G/XFEM. The domain integral calculations are based on the same integration elements used for the computation of the stiffness matrix of the original problem. Discontinuities of the integrands across crack surfaces and across computational element boundaries are fully accounted for. The proposed approach is implemented in the GFEM using a domain form of the $J$-integral and an element-by-element integration scheme like in the standard FEM. The proposed strategy facilitates automatic definition of extraction domains and facilitates control of integration errors since the integrand is a continuous function within each integration element. While we focus on the implementation of the $J$-integral from GFEM solutions, the proposed high-order formulation can be used with, e.g., the FEM and several meshfree methods proposed in the literature. The proposed implementation strategy can also be used with other extraction techniques like those based on the CFM or CIM.

The outline of this paper is as follows. In the following section, the derivation of high-order surface and domain integral methods to compute the $J$-integral are introduced. Section 3 describes the proposed implementation strategy for the domain integral method. A brief summary of the GFEM is also presented in that section along with the proposed auxiliary $q$-function and an approach to automatically define extraction

domains. Section 4 includes several numerical examples to demonstrate the convergence and accuracy of the $J$-integral obtained using the proposed high-order formulation and implementation. Solutions of modeI and mixed mode benchmark fracture mechanics problems are presented along with comparisons to the available numerical solutions from the literature. Section 5 summarizes the main conclusions from this work.

\section{A High-Order Surface and Domain Integral Methods for Three- Dimensional Cracks}

\subsection{Surface Version of the High-Order J-integral}

Crack front parameters in 3-D problems are computed along the crack front. If we consider a point $s$ along a crack front, the $J$-integral can be computed utilizing a contour path enclosing this point. Energy release rate or $J$-integral (used interchangeably herein) is stated as a vector integral $\left(J_{k}, k=1,2,3\right)$ at a point $s$ along the crack front Li et al. [1985], Moran and Shih [1987], Shih et al. [1986]. 


$$
J_{k}(s)=\lim _{\Gamma \rightarrow 0} \int_{\Gamma(s)} H_{k j} n_{j} d C \quad k=1,2,3
$$

where $\Gamma(s)$ is a contour surrounding a three-dimensional crack front and $J_{k}(s), k=1,2,3$ is the pointwise value of crack tip integral per unit advance of the crack front in the direction of crack front unit vectors (tangential, normal or bi-normal), respectively and $\boldsymbol{n}$ is the outward normal vector to the contour path. We consider a crack front coordinate system with normal $\left(x_{1}\right.$ or $\xi$ ), binormal $\left(x_{2}\right.$ or $\left.\zeta\right)$, and tangent $\left(x_{3}\right.$ or $\left.\eta\right)$ as also shown in Figure 1. The energy momentum tensor also referred as Eshelby's tensor Moran and Shih [1987], is given as follows:

$$
H_{k j}=W \delta_{k j}-\sigma_{i j} u_{i, k}
$$

where $W$ is the strain energy density, $\boldsymbol{\delta}$ is the kronecker delta tensor, $\boldsymbol{\sigma}$ is the Cauchy stress tensor, and $\boldsymbol{u}$ is the displacement vector.

In three dimensional problems, pointwise value of the energy release rate is often required to compute the magnitude of crack front advance. This can be computed due to a unit crack growth in the direction of a vector $v$ defined at the crack front. We consider $m_{j}=-n_{j}$ along the contour $\Gamma(s)$ in the following equation to be compatible with the derivations in the literature [Moran and Shih, 1987]:

$$
J(s)=J_{k}(s) v_{k}(s)=\lim _{\Gamma \rightarrow 0}-\int_{\Gamma(s)} H_{k j} m_{j} v_{k}(s) d C
$$
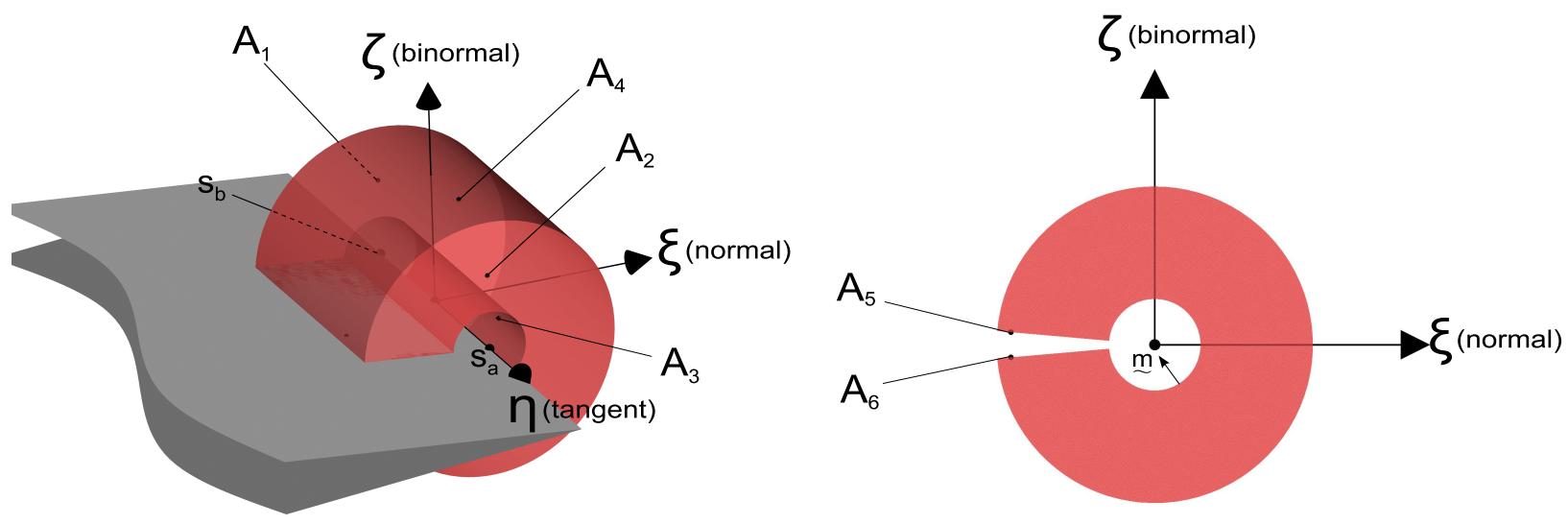

Figure 1: A cylindrical extraction volume to compute J-integral with crack front coordinate system.

The surface integral approach computes the weighted mean of the $J$-integral over a front segment of a 3$\mathrm{D}$ crack front. This assumption is acceptable when the crack front segment $\Gamma_{c}$ (defined between $s_{a}<s<s_{b}$ ) is sufficiently small. An alternative to this approach is to use a polynomial approximation for the $J$-integral, which is, for smooth loading and smoothly shaped crack fronts, a smooth function along the whole crack front Andersson et al. [1995]. An approximation of $J(s)$ is given by $J_{h}(s)$ based on Legendre polynomials in the following equation:

$$
J_{h}(s)=\sum_{\alpha=0}^{N} J_{\alpha} L_{\alpha}(s)
$$


where $L_{\alpha}(s), \alpha=0, \cdots, N$ are Legendre polynomials defined along the crack front line and $J_{\alpha}, \alpha=0, \cdots, N$ are unknown coefficients. It can be realized that the first term of the approximation is the constant term ( since $L_{0}(s)=1$ ) which is also computed using the standard implementation of the $J$-integral as demonstrated below. This approximation removes the restriction for the weighted mean value of the $J$-integral and allows for defining a high-order $J$-integral varying along the crack front, as described next.

The weighted residual method is used to extract the $J$-integral from Equation (3). A residual $r(s)$ is defined between the exact $J$-integral and the approximation of the $J$-integral as follows:

$$
\begin{aligned}
r(s) & =J_{h}(s)-J(s) \\
& =J_{h}(s)+\lim _{\Gamma \rightarrow 0} \int_{\Gamma(s)} H_{k j} m_{j} v_{k}(s) d C
\end{aligned}
$$

Employing the method of weighted residuals (by multiplying the residual by a weight function $w_{\beta}(s)$ and integrating along a crack front segment):

$$
\int_{\Gamma_{c}} r(s) w_{\beta}(s) d s=0 \quad \beta=0, \cdots, N
$$

where the weight function is also formed with the Legendre polynomials as follows:

$$
w_{\beta}(s)=w(s) L_{\beta}(s) \quad \beta=0, \cdots, N
$$

where $w(s)$ is a scalar weight function defined along the crack front and its value is zero at $s=s_{a}$ and at $s=s_{b}$.

Using the definition of the residual given in Equation (5) and the weight function in Equation (7), we can rewrite Equation (6) in the following form:

$$
\int_{\Gamma_{c}}\left[J_{h}(s)+\lim _{\Gamma \rightarrow 0} \int_{\Gamma(s)} H_{k j} m_{j} v_{k}(s) d C\right] w_{\beta}(s) d s=0 \quad \beta=0, \cdots, N
$$

We can substitute $J_{h}$ defined in Equation (4) in the above equation as follows:

$$
\sum_{\alpha=0}^{N}\left(\int_{\Gamma_{c}} L_{\alpha}(s) w_{\beta}(s) d s\right) J_{\alpha}=-\lim _{\Gamma \rightarrow 0} \int_{A_{3}} H_{k j} m_{j} v_{k}(s) w_{\beta}(s) d s
$$

where $A_{3}$ is the inside surface of the tubular domain shown in Figure 1 defined using $\Gamma_{c}$ and $\Gamma(s)$.

Equation (9) can be rewritten in a compact form as:

$$
\sum_{\alpha=0}^{N} A_{\beta \alpha} J_{\alpha}=F_{\beta}
$$

where

$$
\begin{aligned}
A_{\beta \alpha} & =\int_{\Gamma_{c}} L_{\alpha}(s) w_{\beta}(s) d s \\
& =\int_{\Gamma_{c}} L_{\alpha}(s) L_{\beta}(s) w(s) d s
\end{aligned}
$$


and

$$
\begin{aligned}
F_{\beta} & =-\lim _{\Gamma \rightarrow 0} \int_{A_{3}} H_{k j} m_{j} v_{k}(s) w_{\beta}(s) d S \\
& =-\lim _{\Gamma \rightarrow 0} \int_{A_{3}} H_{k j} m_{j} v_{k}(s) L_{\beta}(s) w(s) d S
\end{aligned}
$$

The solution to Equation (10) yields the coefficients of the $J$-integral approximation given by Equation (4). Once the coefficients are obtained, then the $J$-integral can be computed at any location along the crack front. In addition, the $J$-integral obtained using the approximation defined in Equation (4) is a smooth function. It can also be noted from Equation (9) that when the number of the Legendre polynomials is chosen as 1 , we arrive at the following form of the integral:

$$
J_{0}=\frac{-\lim _{\Gamma \rightarrow 0} \int_{A_{3}} H_{k j} m_{j} v_{k}(s) w(s) d S}{\int_{\Gamma_{c}} w(s) d s}
$$

which represents a weighted mean of $J(s)$ along $\Gamma_{c}$. If we take $w(s)=\bar{w}_{k}(s) v_{k}(s)$ (then $\bar{w}_{k}(s)=w(s) v_{k}(s)$ ), the standard definition of the $J$-integral can be recovered Moran and Shih [1987] as follows:

$$
J_{0}=\frac{-\lim _{\Gamma \rightarrow 0} \int_{A_{3}} H_{k j} m_{j} \bar{w}_{k}(s) d S}{\int_{\Gamma_{c}} \bar{w}_{k}(s) v_{k}(s) d s}
$$

\subsection{Domain Version of the High-Order J-integral}

The surface formulation of the $J$-integral described in the previous section is not naturally suited for a three dimensional FE implementation. An alternative form is to define a domain version of the high-order $J$ integral. The domain version is computed in a volume enclosing the whole or a portion of the crack front as shown in Figure 1. Derivations of the domain version of the high-order domain integral stem from the Eshelby's tensor or energy flux tensor $H_{k j}$ similar to those described in Moran and Shih [Moran and Shih, 1987].

Let

$$
H_{k j, j}=b_{k} \quad \text { in } V
$$

where $V$ is any domain enclosing the whole or a portion of the crack front.

A similar procedure to the high-order $J$-integral derivations of the surface version is followed. First, a three-dimensional weight function, $q_{\beta k}$, is defined as

$$
q_{\beta k}(\boldsymbol{x})=q_{k}(\boldsymbol{x}) L_{\beta}(s) \quad \beta=0, \cdots, N
$$

where $L_{\beta}(s), \beta=0, \ldots, N$, are Legendre polynomials.

Functions $q_{\beta k}$ are similar to the function $q_{k}$ defined for the domain version of the standard $J$-integral Moran and Shih [1987]. It possesses the same properties as $q_{k}$ at the boundary $\partial V$ and within the domain 
$V$. The auxiliary function $q_{k}(\boldsymbol{x})$ is a smoothly varying vector field defined as follows:

$$
q_{k}(\boldsymbol{x})= \begin{cases}\bar{w}_{k}(s)=w(s) v_{k}(s) & \text { on } A_{3} \\ 0 & \text { on } A_{1} \cup A_{2} \cup A_{4} \\ \text { otherwise arbitrary } & \end{cases}
$$

We can also define an auxiliary scalar function $q(\boldsymbol{x})$ as

$$
q(\boldsymbol{x})=q_{k}(\boldsymbol{x}) v_{k}
$$

When the point $x$ is on the crack front, this weight function reduces to the following form:

$$
\begin{aligned}
q_{\beta k}(s) & =q_{k}(s) L_{\beta}(s) \\
& =\bar{w}_{k}(s) L_{\beta}(s) \\
& =w(s) v_{k}(s) L_{\beta}(s)=w_{\beta}(s) v_{k}(s)
\end{aligned}
$$

Now, multiplying the high-order weight functions by Equation (15) and integrating over a domain $V$ :

$$
\int_{V}\left(H_{k j, j} q_{\beta k}(\boldsymbol{x})-b_{k} q_{\beta k}(\boldsymbol{x})\right) d V=0 \quad \beta=0, \cdots, N
$$

Using the divergence theorem, we arrive at the following form:

$$
-\int_{V}\left(H_{k j} q_{\beta k, j}+b_{k} q_{\beta k}\right) d V+\int_{\partial V} H_{k j} q_{\beta k} m_{j} d S=0 \quad \beta=0, \cdots, N
$$

where $\partial V=A_{1} \cup A_{2} \cup A_{3} \cup A_{4} \cup A_{5} \cup A_{6}$ as shown in Figure 1. The weight functions vanish on $A_{1} \cup A_{2} \cup A_{4}$. If we assume forward crack front advance and planar crack surface within $V$, we can also eliminate the surface integral contributed by the crack surfaces $A_{5} \cup A_{6}$. Then, we are only left with the contributions from the inner surface of the tube $\left(A_{3}\right)$. Recalling the projection of the weight function along the crack front (Equation (19)), we can simplify the above equation as follows:

$$
\begin{aligned}
& -\int_{V}\left(H_{k j} q_{\beta k, j}+b_{k} q_{\beta k}\right) d V+\int_{A_{3}} H_{k j} q_{\beta k} m_{j} d S \\
= & -\int_{V}\left(H_{k j} q_{\beta k, j}+b_{k} q_{\beta k}\right) d V+\int_{A_{3}} H_{k j} w_{\beta}(s) v_{k}(s) m_{j} d S \\
= & -\int_{V}\left(H_{k j} q_{\beta k, j}+b_{k} q_{\beta k}\right) d V+\lim _{\Gamma \rightarrow 0} \int_{A_{3}} H_{k j} w_{\beta}(s) v_{k}(s) m_{j} d S=0 \quad \beta=0, \cdots, N
\end{aligned}
$$

where the second term on the left hand side can be recalled from the surface version of the high-order $J$-integral (Equation (9))

$$
\begin{aligned}
& -\int_{V}\left(H_{k j} q_{\beta k, j}+b_{k} q_{\beta k}\right) d V+\lim _{\Gamma \rightarrow 0} \int_{A_{3}} H_{k j} w_{\beta}(s) v_{k}(s) m_{j} d S \\
= & -\int_{V}\left(H_{k j} q_{\beta k, j}+b_{k} q_{\beta k}\right) d V-\sum_{\alpha=0}^{N}\left(\int_{\Gamma_{c}} L_{\alpha}(s) w_{\beta}(s) d s\right) J_{\alpha}=0
\end{aligned}
$$


Similar to the surface version of the high-order $J$-integral, the same compact form introduced in Equation (10) can be used to represent the terms appearing in Equations (24) and (25). In the current implementation for high-order domain integrals, only straight crack fronts are considered. Therefore, crack front vector $v_{k}$ is assumed constant in the following integrals

$$
\begin{gathered}
F_{\beta}=-\int_{V}\left[H_{k j} q_{\beta k, j}+b_{k} q_{\beta k}\right] d V \\
=-\int_{V}\left[H_{k j}\left(q, j v_{k} L_{\beta}+q v_{k} L_{\beta, j}\right)+b_{k} q_{\beta k}\right] d V \\
A_{\beta \alpha}=\int_{\Gamma_{c}} L_{\alpha}(s) w_{\beta}(s) d s \\
=\int_{\Gamma_{c}} L_{\alpha}(s) L_{\beta}(s) w(s) d s
\end{gathered}
$$

Similarly, when the number of the Legendre polynomials are chosen as $1\left(L_{0}=1\right)$ and if we take $w(s)=$ $\bar{w}_{k}(s) v_{k}(s)$, then the standard form of the domain version of the $J$-integral is recovered $\left(q_{0 k}=q_{k}\right.$ and $J_{h}(s)=$ $\left.J_{0}\right)$ as follows:

$$
J_{0}=\frac{-\int_{V}\left[H_{k j} q_{k, j}+b_{k} q_{k}\right] d V}{\int_{\Gamma_{c}} \bar{w}_{k}(s) v_{k}(s) d s}
$$

If the Eshelby's tensor is assumed to be divergence free, the body force term defined by $b_{k}$ cancels in Equation (24). This assumption is true when there are no body forces, residual stresses and thermal stresses [Moran and Shih, 1987]. The high-order formulation of the $J$-integral presented in this section is fully compatible with partition of unity based methods (GFEM and XFEM). This approach can provide smoothly varying crack front parameters such as the $J$-integral, which might be an issue for the computation of crack front advance in these methods.

\section{Numerical Implementation Strategy}

The numerical implementation of the domain integral is done using the GFEM. The GFEM enables modeling of arbitrarily located cracks within a FE mesh and alleviates the mesh design issues arising in fracture problems, particularly in 3-D. Some important aspects of the GFEM is revisited along with a very brief introduction. Subsequently, the numerical implementation of the high-order domain integral in the GFEM is presented.

\subsection{A Brief Summary of Generalized Finite Element Method}

The generalized FEM is an instance of the so-called partition of unity [Babuška et al., 1994, Melenk and Babuška, 1996, Duarte and Oden, 1996b,a] based methods. Some meshless or meshfree methods are also from the same family of partition of unity based methods. A partition of unity based approximation of a scalar scalar field $u(\boldsymbol{x})$ can be expressed with partition of unity shape functions and local 
approximations as:

$$
u_{h}(\boldsymbol{x})=\sum_{\alpha=1}^{N} \varphi_{\alpha}(\boldsymbol{x}) u_{h \alpha}(\boldsymbol{x})
$$

where $\varphi_{\alpha}$ constitute a partition of unity $\left(\sum_{\alpha=1}^{N} \varphi_{\alpha}(\boldsymbol{x})=1\right.$ for all $\boldsymbol{x}$ in the domain of analysis $\left.\Omega\right), \alpha$ is a node in the finite element mesh and $u_{h \alpha}(\boldsymbol{x}), \alpha=1, \ldots, N$, are local approximations. In the GFEM, the partition of unity is provided by low order Lagrangian shape functions. A local approximation $u_{h \alpha}(\boldsymbol{x})$ is given by

$$
u_{h \alpha}(\boldsymbol{x})=\sum_{i=1}^{D_{L}} a_{i \alpha} L_{i \alpha}(\boldsymbol{x}) \quad(\text { no summation on } \alpha)
$$

where $a_{i \alpha}$ and $L_{i \alpha}, i=1, \ldots, D_{L}$, are nodal coefficients and enrichment functions, respectively. The function $u_{h \alpha}(\boldsymbol{x})$ is a local approximation of the field $u(\boldsymbol{x})$ defined on the support of partition of unity function $\varphi_{\alpha}$. This support (also called a cloud), $\omega_{\alpha}$, denotes a subdomain where the partition of unity function for an arbitrary node $\alpha$ is nonzero. Local approximations belong to the local spaces as follows:

$$
\chi_{\alpha}\left(\omega_{\alpha}\right)=\operatorname{span}\left\{L_{i \alpha}(\boldsymbol{x})\right\}_{i=1}^{D_{L}}
$$

Details of the theoretical background of the GFEM and partition of unity based methods are described in [Babuška et al., 1994, Melenk and Babuška, 1996, Duarte and Oden, 1996b,a, Duarte et al., 2000, 2001 ]. The enrichment functions are selected such that:

$$
L_{1 \alpha}=1
$$

We can rewrite the partition of unity approximation defined in Equation (27) using the local approximation given by Equation (28) as follows:

$$
u_{h}(\boldsymbol{x})=\sum_{\alpha=1}^{N} \varphi_{\alpha}(\boldsymbol{x}) \sum_{i=1}^{D_{L}} a_{i \alpha} L_{i \alpha}(\boldsymbol{x})=\sum_{\alpha=1}^{N} \sum_{i=1}^{D_{L}} a_{i \alpha} \phi_{i \alpha}(\boldsymbol{x})
$$

where $\phi_{i \alpha}(\boldsymbol{x})$ is called a generalized finite element function and is defined from the product of the partition of unity and a local enrichment function in the following form:

$$
\phi_{i \alpha}(\boldsymbol{x})=\varphi_{\alpha}(\boldsymbol{x}) L_{i \alpha}(\boldsymbol{x}) \quad(\text { no summation on } \alpha)
$$

The partition of unity functions in Equation (32) $\left(\varphi_{\alpha}, \alpha=1, \ldots, N\right)$ in the current implementation of the GFEM are linear Lagrangian finite element shape functions. Some other examples of partition of unity functions are functions derived from moving least squares methods and Shepard functions [Duarte and Oden, 1996a] as they are used in meshless methods.

\subsection{Numerical evaluation of the high-order domain integral}

The numerical implementation of domain integrals in G/XFEM exhibits various challenges since their computation can not be performed using the computational elements in a nicely structured mesh as in the FEM. Another difficulty is the approximation of the scalar auxiliary function ( $q$-function) representing a virtual crack advance. These challenges along with the implementation details of the high-order domain integral formulations are discussed next. 
In Section 2, the derivations of a high-order and standard formulations for the computation of the $J$ integral are described. Both approaches are implemented in the GFEM. Numerical implementation of the high-order (Equations (24) (25)) and the standard $J$-integral given by Equation (26) slightly differ from each other only in the selection of extraction domains. Numerical implementations for the line integration and volume integrals appearing in these equations are described in this section.

\subsubsection{Construction of extraction domains}

The integration domain for the computation of the $J$-integral given by Equations (24) or (26) is defined by layers of elements enclosing the crack front. This volume is formed by adding layers of elements at selected locations along the crack front where the $J$-integral needs to be calculated. These elements are computational elements in which element solution vector was computed during the GFEM analysis. Stresses, displacement vector, and strain energy density from the GFEM solution can be directly used in the evaluation of the integral appearing in Equation (24) or Equation (26) after transforming these variables to the crack front coordinate system.

Construction of integration domain differs for the standard and high-order $J$-integral implementation. The high-order $J$-integral approach employs a single or a few extraction domains enclosing the entire crack front whereas the standard $J$-integral is computed in a piecewise manner using sufficiently small extraction domains. The crack front in the GFEM is discretized by segments as shown in Figure 2. Each segment is composed of two crack front vertices. In order to constitute an integration domain for the standard $J$-integral approach, a fraction of the crack front formed by two segments and three vertices are first selected. A crack front coordinate system is chosen to be at a point in the middle of a straight line connecting the beginning and end vertices. This point is identically equal to the middle vertex for straight crack fronts. The entire crack front should be discretized into sufficiently small segments for curved crack fronts in order not to introduce errors due to crack front geometry approximations. Then, the elements enclosing this portion of the crack front (formed by two segments) is automatically added to a list, layer by layer. Finally, a bounding box is defined to prevent outgrowing of the domain in all directions (normal, tangent and binormal at the crack front). The elements outside this bounding box are removed from the list. The use of a bounding box is particularly needed to trim the elements in the tangential direction so that a domain enclosing only two crack front segments can be obtained. This procedure is repeated along the crack front to compute the $J$-integral pointwise at each vertex except at end vertices of surface breaking cracks.

The implementation of the high-order $J$-integral selects all or a large fraction of the crack front segments and builds a domain around the entire crack front at once. In the high-order implementation the extraction domain may be composed of a single large domain whereas in standard domain integral requires the construction of multiple extraction domains that sweep the entire crack front. Once the extraction domain is constructed, the computation of the energy release rate is performed element by element using integration elements as discussed in the next section.

\subsubsection{Computation of volume integral $\left(F_{\beta}\right)$}

The pointwise value of the $J$-integral can be composed of components in the directions of crack front coordinate system as shown in Equation (3). Each component represents the value of the energy released for an advance of the crack front in one of the crack front directions (normal, bi-normal, or tangential). In order to simplify the implementation, only one component of the $J$-integral is considered in this study. Numerical implementation is carried out assuming $k=1$, hence considering only crack front advance in 


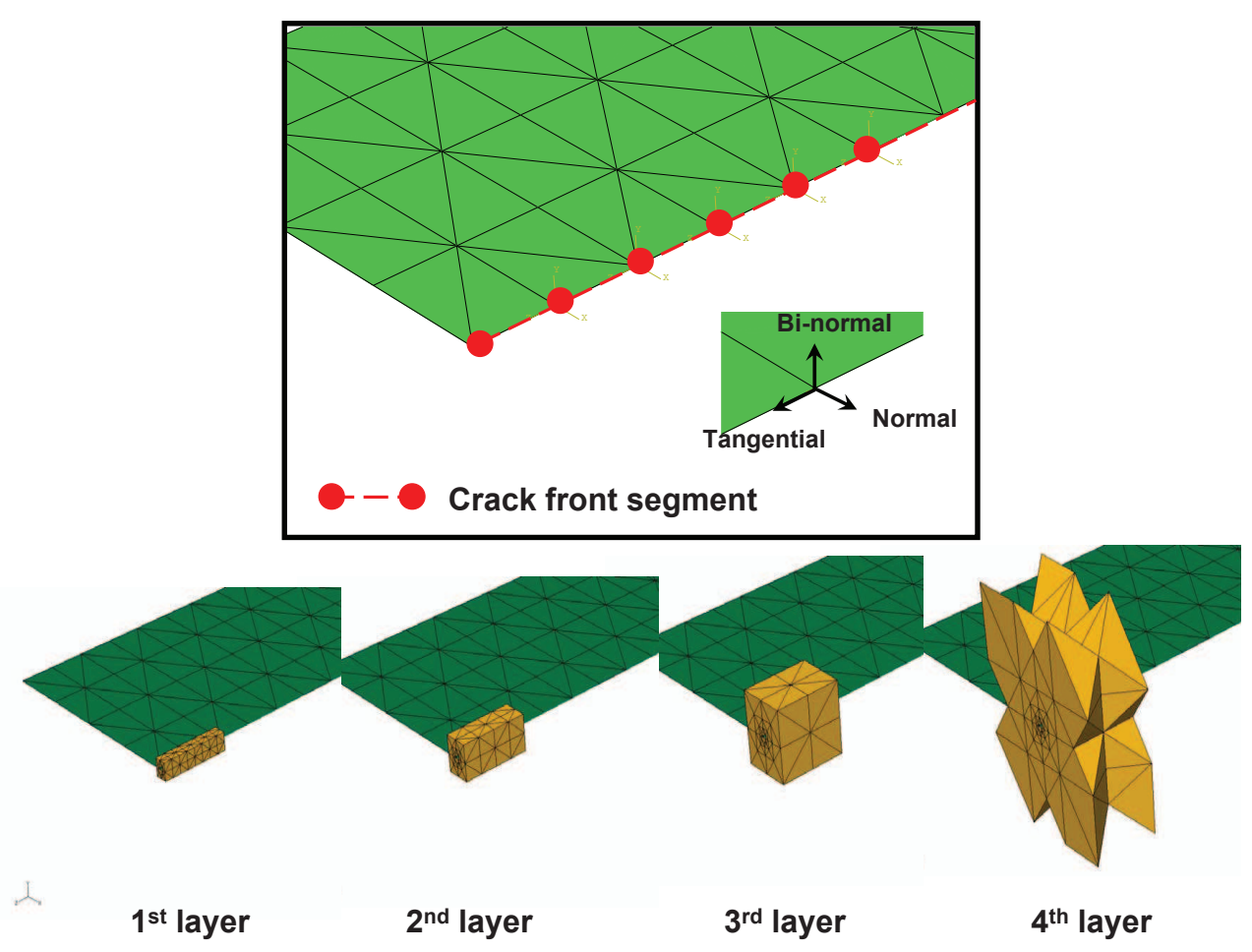

Figure 2: Crack front geometry and selection of the extraction domains for the standard domain integral method.

the normal direction. This component of the $J$-integral is associated with a unit crack front advance in the normal direction $x_{1}$ as shown by the crack front coordinate system in Figure 1. In the absence of body forces, crack surface tractions, and with the assumption of crack front extension only in the normal direction, the $J$-integral reduces to a simplified volume integral.

The volume integral appearing in Equation (24) or in the numerator of Equation (26) is an integral defined over a domain enclosing a specified portion of the crack front (composed of two segments as discussed in the previous section) or the whole crack front for the high-order $J$-integral implementation. Since this domain is the union of finite elements used in the computation of the stiffness matrix, solution variables such as displacements, stresses, strains, etc. can readily be accessed. The auxiliary q-function introduced in Section 2.2, however, needs to be defined. The q-function is approximated using partition of unity functions (in this case linear tetrahedral shape functions). Assuming a crack front advance only in the normal direction $\left(q_{\beta} \equiv q_{\beta 1}\right)$, the $q$-function can be defined as follows:

$$
q_{\beta}(\xi, \eta, \zeta)=\sum_{\alpha=1}^{4} \varphi_{\alpha}(\xi, \eta, \zeta) \hat{q}_{\alpha} L_{\beta}(s) \quad s \in \Gamma_{c}, \quad \beta=0, \cdots, N
$$

where $\hat{q}_{\alpha}$ is the value of $q$-function at node $\alpha$ and $\varphi_{\alpha}$ is a linear finite element shape function. The nodal values of the q-function are assigned to nodes automatically during the formation of the extraction domains. The values vary from 1 at the crack front to 0 at the outside boundaries of the extraction domain. A twodimensional version of the q-function defined above was reported in [Moës et al., 1999]. The Legendre polynomials are added to this equation for the computation of the high-order $J$-integral. This requires 
mapping from the natural coordinate system of an integration element $(\xi, \eta, \zeta)$ to the crack front coordinate $s$ to compute the contribution of the Legendre polynomial.

The auxiliary q-function does not have a closed-form mathematical expression. It can vary from domain to domain depending on the crack front refinement level. The effect of different q-functions on the computed fracture parameters was studied and it has been shown that the $J$-integral values computed from moderately sized domains are insensitive to the choice of $q$-function Shih et al. [1986]. Nikishkov and Atluri Nikishkov and Atluri [1987] also used different $q$-functions in the computation of SIF's and obtained acceptable results for various shapes of the q-function defined in the domain.

The smoothness requirement for the $q$-function is satisfied in a piecewise linear sense due to linear finite element shape functions. An example of a $q$-function is shown in Figure 3 for a straight edge crack problem. This figure shows the variation of the q-function in the extraction domain. As shown, values of $q$-function are 0 everywhere at the outside boundaries and vary from 0 to 1 inside the domain.

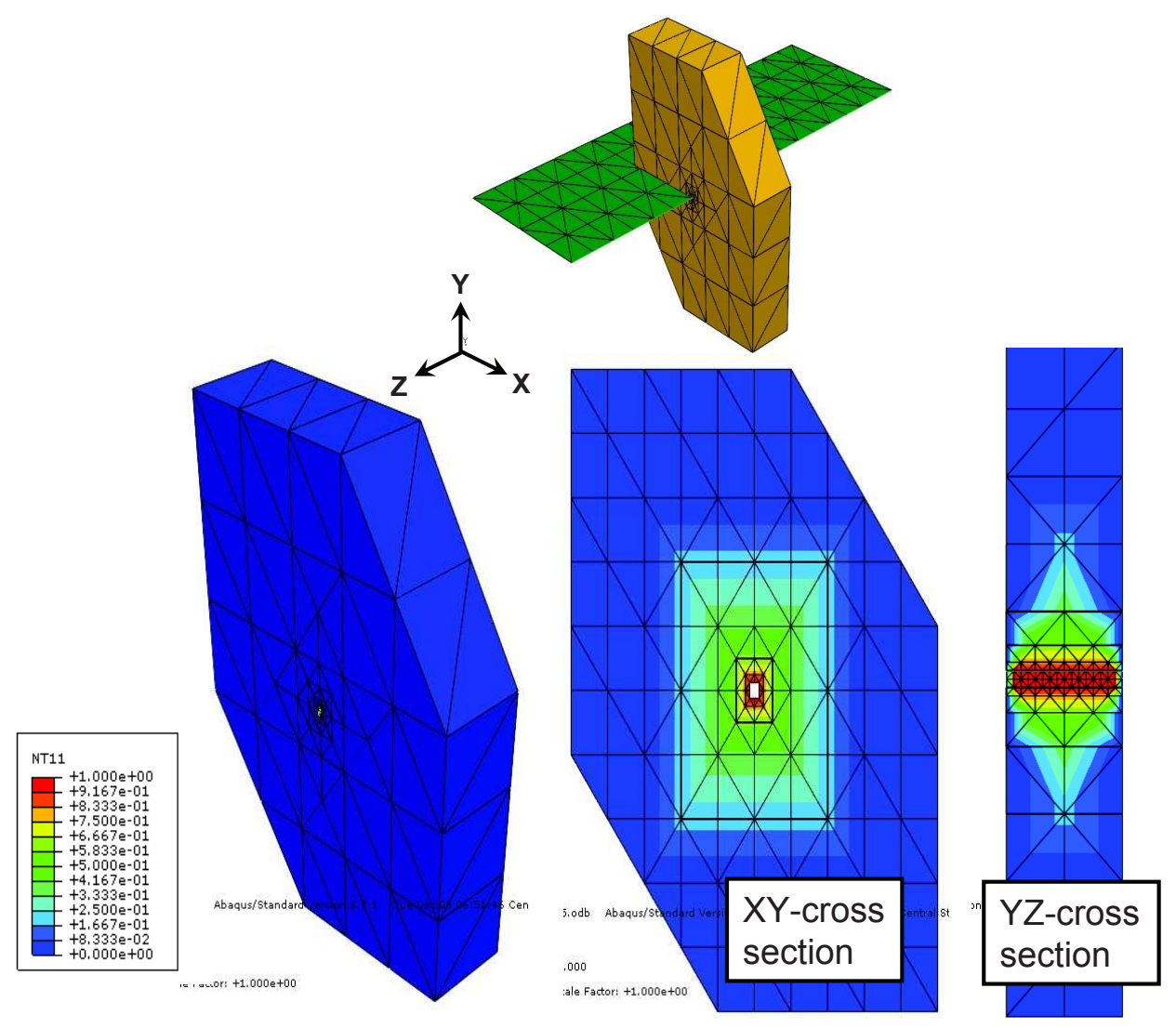

Figure 3: Variation of the q-function in an extraction domain for an edge-crack problem. The order of Legendre polynomials is 0 .

Gauss quadrature is applied to the volume integral defined in Equation (24). Each quantity in this expression needs to be computed at the integration points and then transformed to the crack front coordinate system using transformation matrices built between global and crack front coordinate systems. 


$$
F_{\beta}=-\sum_{k}^{N}\left[W\left(\frac{\partial q}{\partial x_{1}}+q L_{\beta, 1}\right)-\sigma_{i j} \frac{\partial u_{i}}{\partial x_{1}}\left(\frac{\partial q}{\partial x_{j}} L_{\beta}+q L_{\beta, j}\right)\right]_{\xi_{k}} \operatorname{det}[J] w_{k}
$$

where $q \equiv q_{1}$. The terms in the bracket are evaluated at each integration point $\boldsymbol{\xi}_{k}(k=1, \cdots, N) . L_{\beta, 1}=0$ since the Legendre polynomials are one dimensional functions defined along the crack front direction, $\operatorname{det}[J]$ is the determinant of the element Jacobian, and $w_{k}$ is a integration weight.

\subsubsection{Computation of the Line Integral $\left(A_{\beta \alpha}\right)$}

The line integral defined by $A_{\beta \alpha}$ in Equation (25) requires the values of the $q$-function along the crack front. Once the q-function is assigned to the nodes, the values of the q-function at the crack front itself can be computed using the finite element approximation of the q-function in Equation (33). Independent discretizations of the crack surface and the 3-D domain presents a challenge for finding the crack front values of the q-function, since the crack front is not composed of finite element nodes as a part of the finite element discretization. This difficulty is overcome by computing the values of the $q$-function within each element cut by the crack surface. Equation (33) is used in the approximation of the q-function along the crack front. The procedure is illustrated in Figure 4 for the standard $J$-integral implementation. Figure 4 also shows how integration points are chosen at the crack front. The variation of the q-function computed at these integration points is also shown in the same figure for two mesh refinement levels. The curves represent a crack front advance in the normal direction, which are used to compute the line integral that appears in the denominator of Equation (26).
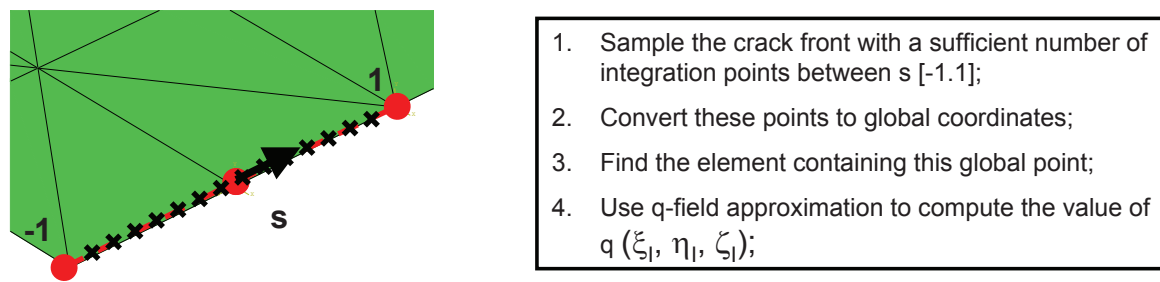

* Integration points $\left(\xi_{1}, \eta_{1}, \zeta_{\mid}\right)$
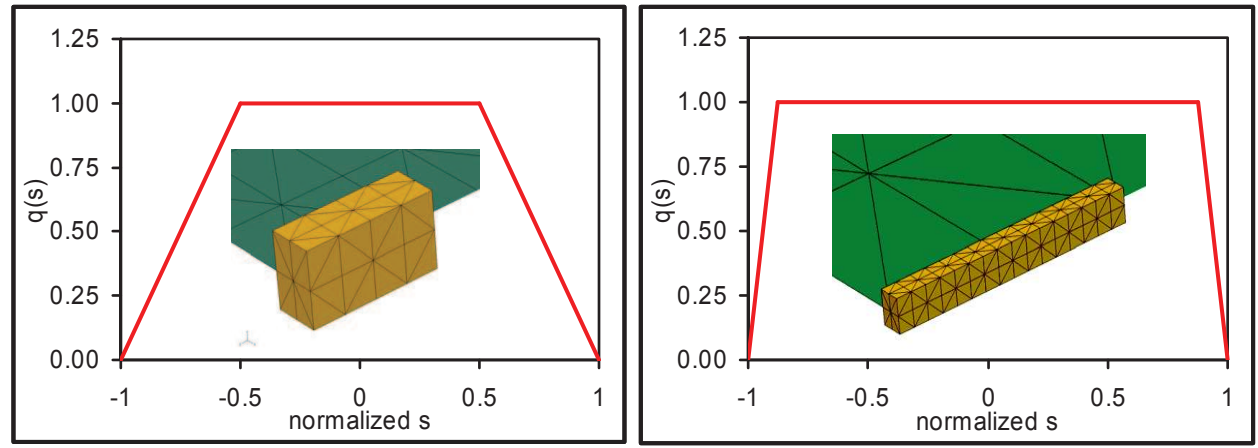

Figure 4: Variation of q-function along the crack front of an edge crack problem using the standard J-integral approach. Crack front values of q-function are shown for two different crack front refinement levels.

On the other hand, the q-function for the high-order implementation is built along the entire or a large fraction of the crack front. Examples of integrands $w(s) L_{\alpha}(s) L_{\beta}(s)$ from Equation (25) are shown in Figure 
5. The integrand is sampled along the crack front. Only diagonal components are shown in the figure. A numerical integration procedure is followed to compute the line integral. The number of integration points used to sample the q-function and the Legendre polynomials are chosen high for accurate computation of the integral.
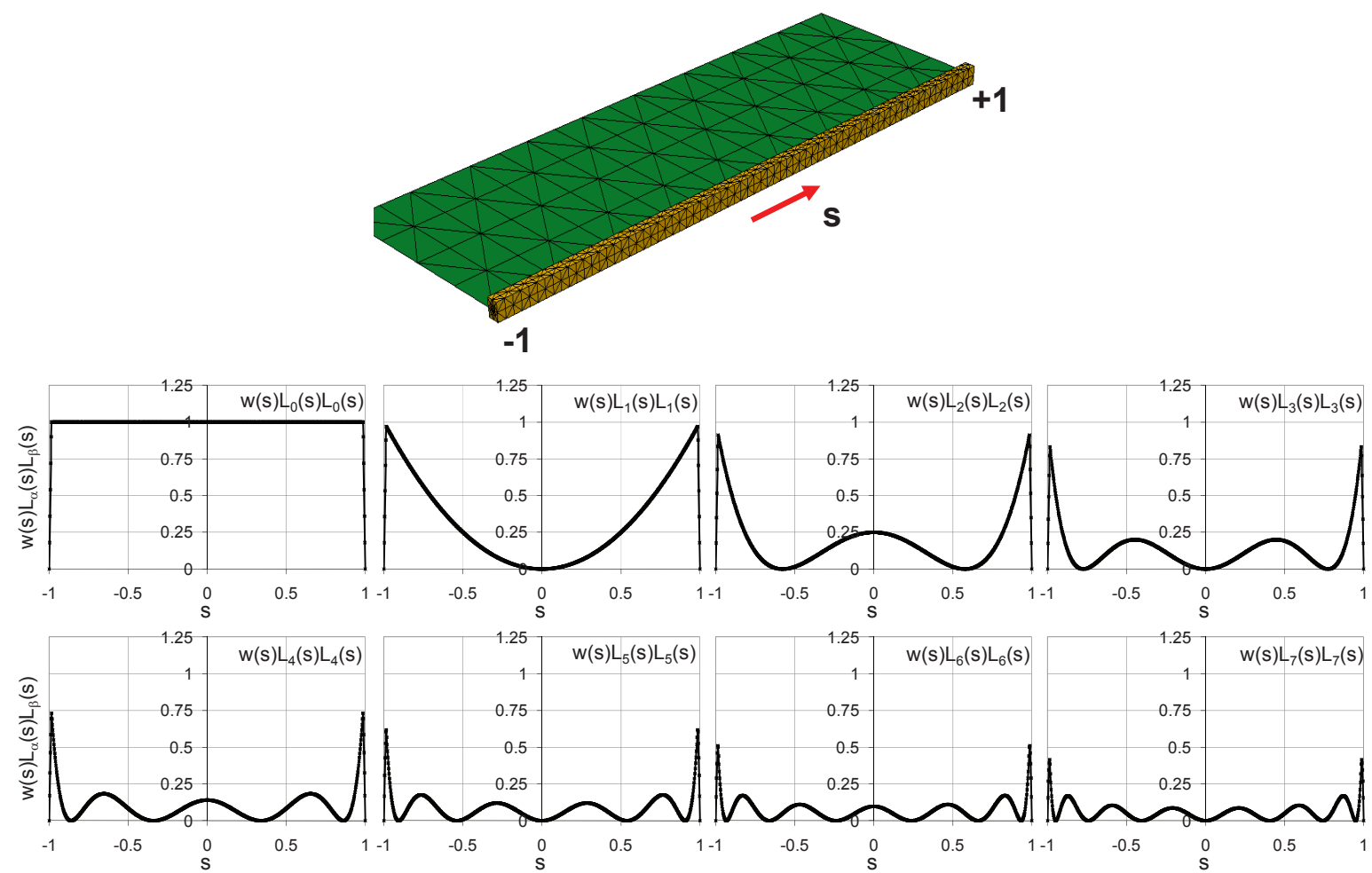

Figure 5: Variation of the integrand from Equation (25) along the crack front of an edge-crack problem using the high-order J-integral approach. In the figure, $\alpha, \beta$ are the degree of the Legendre polynomials.

\section{Numerical Examples}

In this section, we analyze the accuracy of the proposed formulations and implementation approaches to compute the energy release rate. Several benchmark fracture mechanics problems are analyzed in order to investigate accuracy and robustness of the standard and the high-order formulation of the $J$-integral using mode-I and mixed mode examples with straight and curved crack fronts. Convergence analysis is also performed to verify convergence rates of extracted energy release rate.

\subsection{Edge Cracked Bar}

The edge cracked bar is a very commonly used problem in the development and verification of new numerical and mathematical strategies to compute energy release rate and/or stress intensity factors (SIF's). Li et al. [1998] solved this problem using the boundary element method. The results from that study are used as the reference solution herein. Pereira et al. [2009a] solved the same problem using the GFEM with 
the Cut-off Function Method (CFM) and the Contour Integral Method (CIM). A locally refined mesh with increasing polynomial approximation order (we refer to this approach as $h p$-GFEM hereafter) was used in that study and it has been shown that the $h p$-GFEM can provide very accurate results and fast convergence rates for the SIF's. Details can be found in [Pereira et al., 2009b] and [Pereira et al., 2009a].

The edge crack problem with the same boundary conditions and geometry [Li et al., 1998], [Pereira et al., 2009a] is also analyzed in this section. This problem consists of a rectangular bar sliced by a through the thickness crack as shown in Figure 6. The geometry is taken as $h / t=0.875, a / t=0.5$, and $w / t=1.5$. As for the material properties, linear elastic properties are used. Young's modulus and Poisson's ratio are set to 1.0 and 0.3 , respectively. Various local refinement levels are studied to test the robustness of the proposed formulation and implementation for different crack front meshes. Three ratios of crack front element size to crack length, $L_{e} / a$, are used: $L_{e} / a=0.069, L_{e} / a=0.035$ and $L_{e} / a=0.017$. The results obtained using the standard domain integral implementation are referred to as $J_{o}$ in the figures.

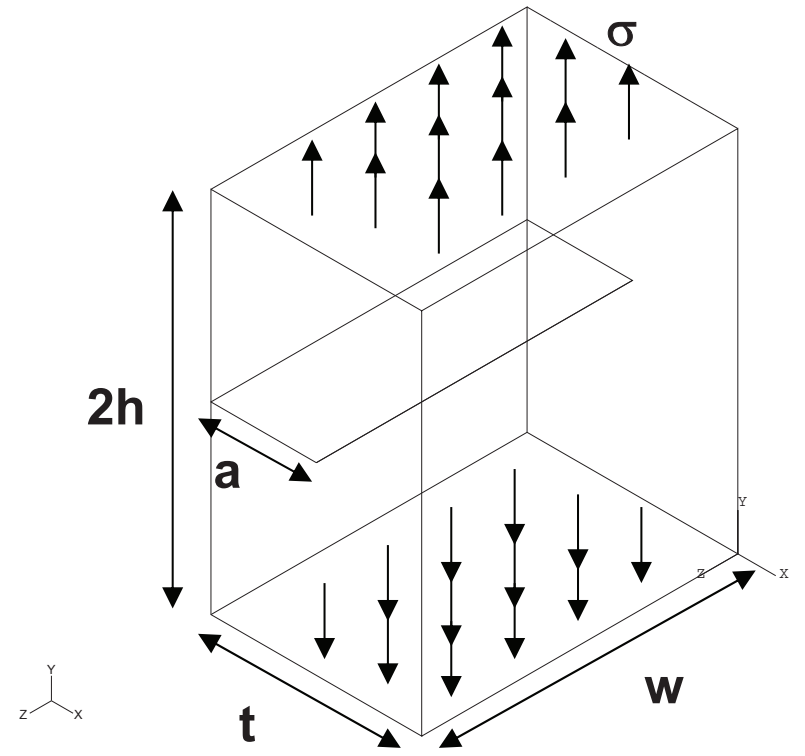

Figure 6: Edge crack bar subjected to uniform tensile tractions.

The extraction domains along the crack front are built using the procedure described in the previous sections. During the computation of $J$-integral with the standard approach, pointwise values of the energy release rate are computed at the vertices along the crack front except at the edges. Figure 7 shows selected extraction domains used in the computation of energy release rate along the crack front. These domains are formed by adding 5 layers of elements to the crack front elements, the first of which is removed later on to create a hollow domain. On the other hand, the high-order implementation can use bigger extraction domains enclosing the whole or a segment of the crack front. 


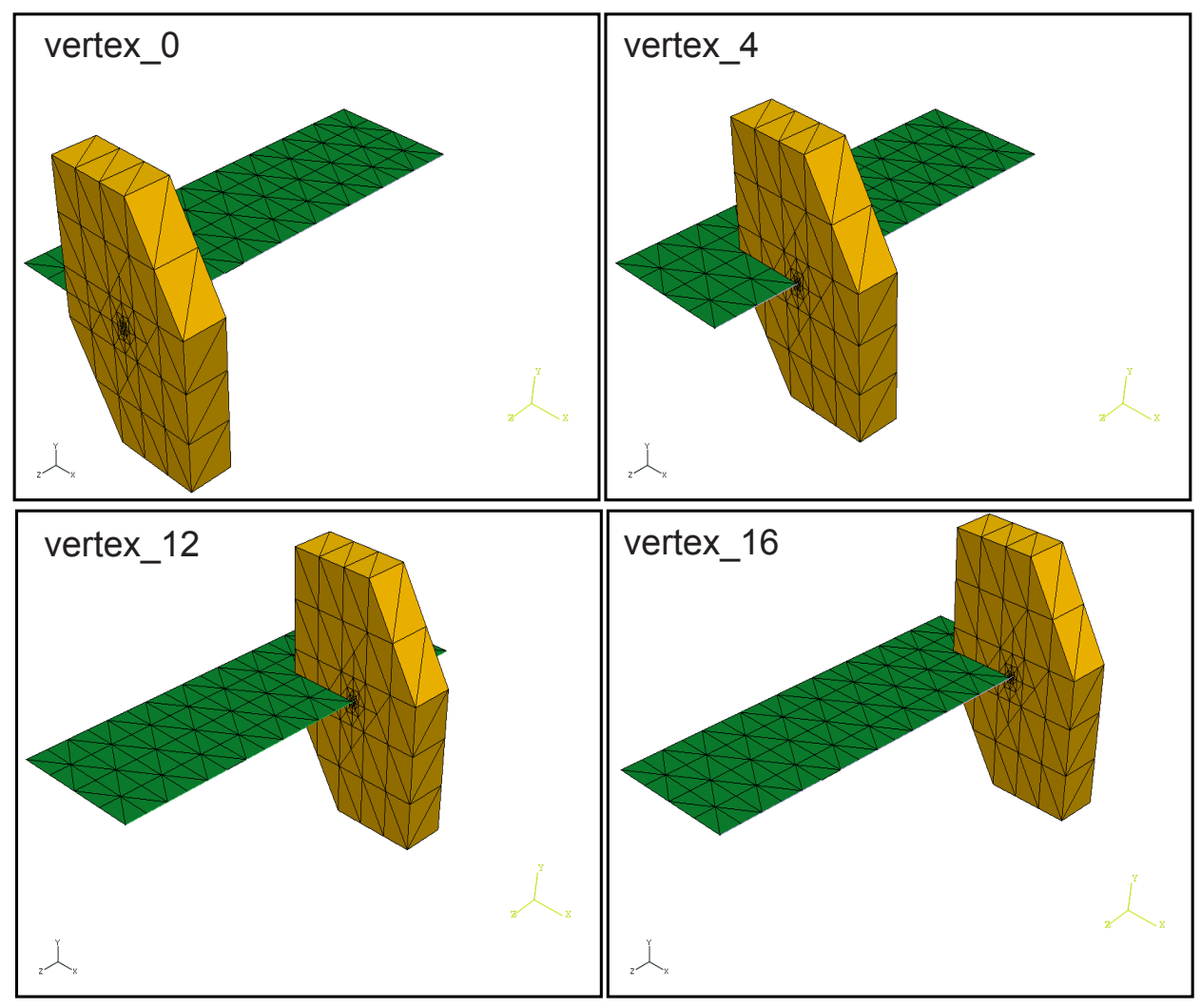

Figure 7: Extraction domains along the crack front at vertices 0, 4, 12, and 16 of an straight edge crack. 
The energy release rate computed along the crack front using the standard formulation of the $J$-integral is shown in Table 1 along with a comparison to the solution obtained by Li et al. [1998]. In order to demonstrate path independency of the implementation, energy release rate computed using various domain sizes are also included in the same table. Energy release rate is computed using domains built with various number of layers (from 1 to 5). In all of these domains, the first layer of elements selected around the crack front is removed from the list of elements to create a hollow domain. The results shown in Table 1 and Figure 8 were extracted from a GFEM solution computed using polynomial approximation order $p=4$. As shown in Figure 8 and Table 1, the results are in good agreement with the reference solution and also verify the path independency of the solution with respect to various domain sizes used in this analysis.

Table 1: Energy release rate values computed along the edge crack front example ( $p=4$ and $\left.L_{e} / a=0.0174\right)$.

\begin{tabular}{|c|c|c|c|c|c|c|c|}
\hline \multirow[t]{2}{*}{ Vertex } & \multirow[t]{2}{*}{$s / w$} & \multicolumn{5}{|c|}{ Number of element layers added to domain } & \multirow[t]{2}{*}{ Ref. Li et al. [1998] } \\
\hline & & 1 & 2 & 3 & 4 & 5 & \\
\hline 0 & -0.5 & \multicolumn{5}{|c|}{ not calculated at this point } & \multirow{18}{*}{0.012052} \\
\hline 1 & -0.444445 & 0.010348 & 0.010355 & 0.010361 & 0.010366 & 0.010370 & \\
\hline 2 & -0.388889 & 0.011293 & 0.011300 & 0.011306 & 0.011311 & 0.011315 & \\
\hline 3 & -0.333333 & 0.011648 & 0.011655 & 0.011661 & 0.011667 & 0.011672 & \\
\hline 4 & -0.277778 & 0.011826 & 0.011834 & 0.011840 & 0.011846 & 0.011850 & \\
\hline 5 & -0.222222 & 0.011922 & 0.011930 & 0.011936 & 0.011942 & 0.011947 & \\
\hline 6 & -0.166666 & 0.011977 & 0.011984 & 0.011991 & 0.011996 & 0.012000 & \\
\hline 7 & -0.111111 & 0.012004 & 0.012012 & 0.012018 & 0.012024 & 0.012029 & \\
\hline 8 & -0.055556 & 0.012019 & 0.012027 & 0.012033 & 0.012038 & 0.012042 & \\
\hline 9 & 0.000000 & 0.012024 & 0.012031 & 0.012037 & 0.012043 & 0.012048 & \\
\hline 10 & 0.055556 & 0.012019 & 0.012027 & 0.012033 & 0.012039 & 0.012042 & \\
\hline 11 & 0.111111 & 0.012004 & 0.012012 & 0.012018 & 0.012024 & 0.012029 & \\
\hline 12 & 0.166667 & 0.011976 & 0.011984 & 0.011990 & 0.011996 & 0.012000 & \\
\hline 13 & 0.222222 & 0.011923 & 0.011930 & 0.011937 & 0.011942 & 0.011948 & \\
\hline 14 & 0.277778 & 0.011828 & 0.011835 & 0.011841 & 0.011846 & 0.011850 & \\
\hline 15 & 0.333333 & 0.011648 & 0.011655 & 0.011662 & 0.011667 & 0.011672 & \\
\hline 16 & 0.388889 & 0.011298 & 0.011306 & 0.011312 & 0.011317 & 0.011320 & \\
\hline 17 & 0.444444 & 0.010345 & 0.010351 & 0.010357 & 0.010362 & 0.010367 & \\
\hline 18 & 0.5 & & not cal & ulated at th & s point & & \\
\hline
\end{tabular}

The same problem is also solved using the high-order formulation of the $J$-integral. Figure 9 shows the results from the solution of the same problem using high-order $J$-integral implementation in a domain enclosing the entire crack front. The order of the Legendre polynomials varies from 1 to 7 in these examples whereas the polynomial order of the GFEM approximation is 4. The results are compared to the reference values obtained from the literature [Li et al., 1998]. As the order of Legendre polynomials increases, the solution improves and approaches the reference values. However, it is important to note the fluctuations in Figure 9 at the center section likely to be caused by the behavior of the $J$-integral near the vertices of the crack front. In order to address this problem and improve the extracted values at the vertices, the crack front domain is partitioned into three separate extraction domains: at the two vertices and at the center. The same high-order domain integral formulations are used to extract energy release rate at these domains. Figure 10 illustrates the results at the center and vertex domains, separately. The extraction domains used to compute the $J$-integral are also shown as inserts to this figure. The order of Legendre polynomials are chosen 


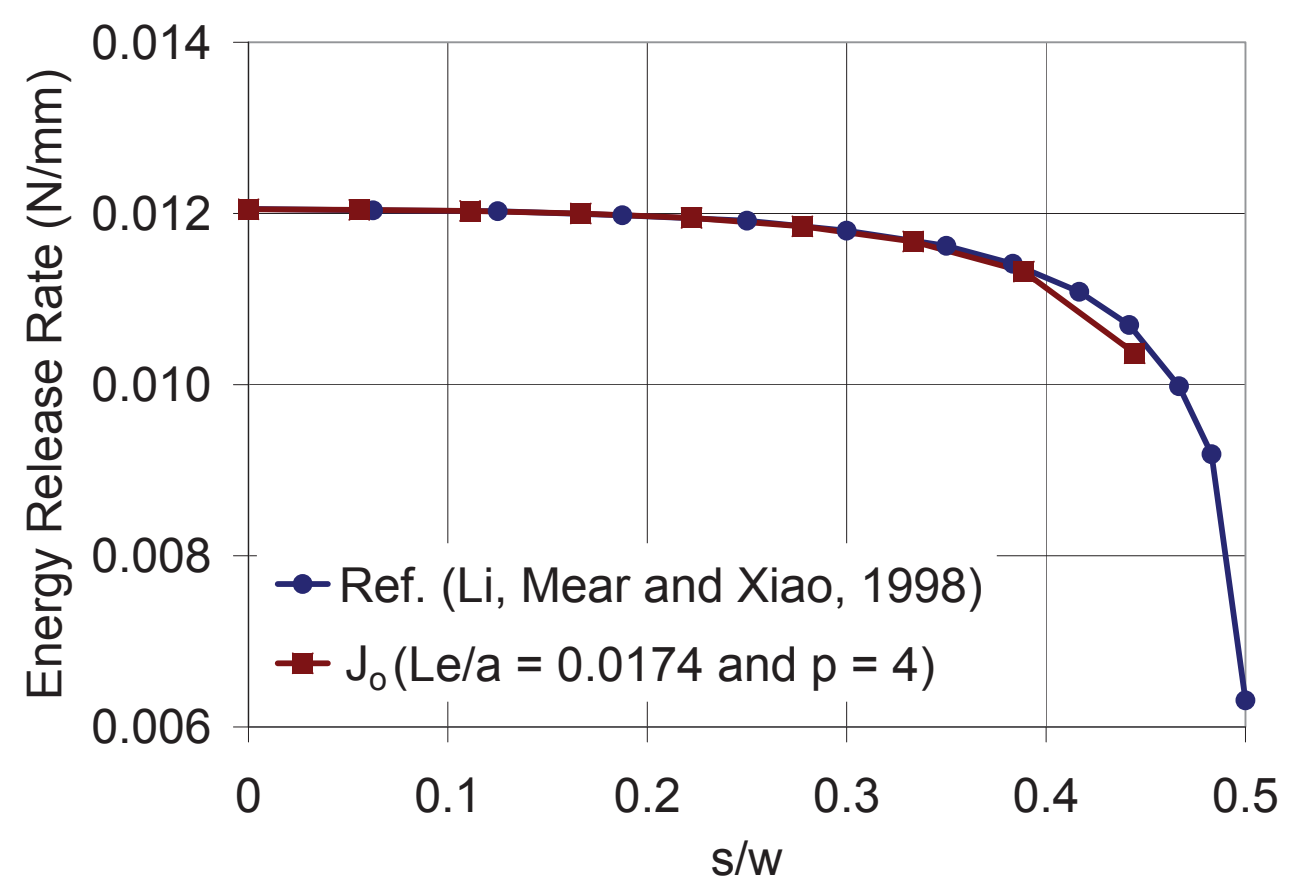

Figure 8: Energy release rates computed using the standard J-integral implementation with the results from a reference solution for the edge crack bar example Li et al. [1998].

compatible with the order of approximation at the center domain. Since the order of approximation is 4 and the domain integral integrand contains the derivatives of the solution, the order of Legendre polynomials is chosen 3 at the center. Higher order Legendre polynomials are used at the vertices to capture the sharp gradients of $J$ at that regions. As shown in Figure 10, a very good match between the reference solution and the high-order domain integral is achieved at the center and at the vertex domains. The boundary layer behavior of the $J$-integral is clearly captured.

Another objective of this example is to check if optimum convergence rates can be recovered using the proposed formulation and implementation of the domain integral method. Convergence analysis with the GFEM was shown to be exponential for strain energy and SIF's Pereira et al. [2009a], in which SIF's were computed using the CIM and CFM in locally refined meshes. Polynomial order of the approximation was increased on strongly graded meshes ( $p$-convergence). A very similar approach is also followed in this study. The mesh is fixed at three crack front refinement levels and the order of the polynomial approximation is increased from $p=1$ to $p=4$. Crack front elements are locally refined to the levels $L_{e} / a$ ratios around $0.069,0.035$, and 0.017 , respectively. The value of the energy release rate computed at the middle of crack front $(s / w=0)$ is used in the convergence analysis. Since there is no analytical solution for the problem at hand, an extrapolation procedure based on a-priori error estimates Szabó and Babuška [1991] is used to compute reference values for strain energy and the energy release rate at $s / w=0$. The extrapolation is performed using three solutions obtained from the finest crack front mesh and approximation orders $p=2,3,4$. Details on the extrapolation procedure are described in Szabó and Babuška [1991]. The computed reference value of strain energy and energy release rate are $U_{\text {ref }}=0.343555489$ and $J_{\text {ref }}(0)=0.01206851$. Figure 11 demonstrates the results from this convergence exercise. The figure shows the relative error in the strain energy and energy release rate with respect to the number of degrees of freedom. The slope of the curves are also illustrated as an insert to Figure 11. Similar convergence 


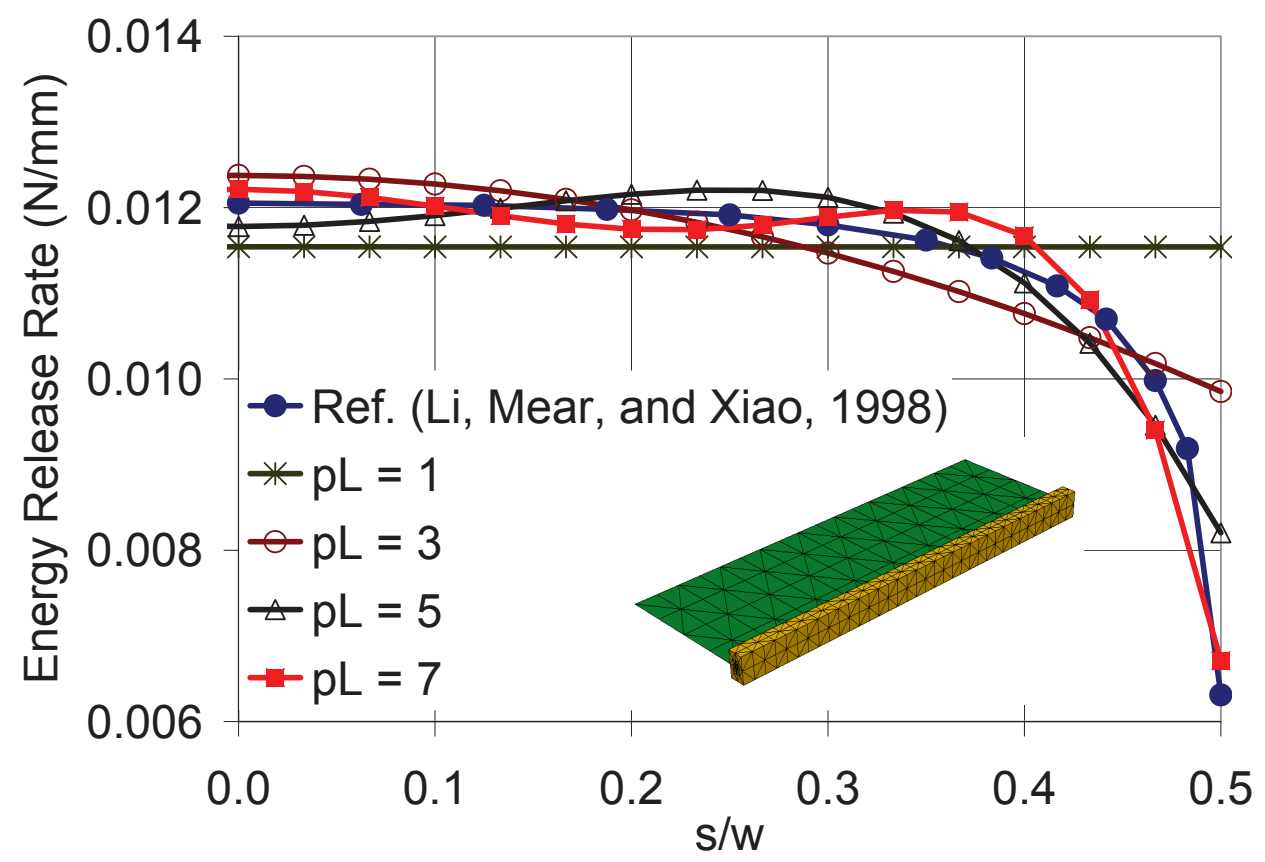

Figure 9: High-order J-integral computed for the edge crack problem using whole crack front domain ( $p L$ : polynomial order of the Legendre polynomials).

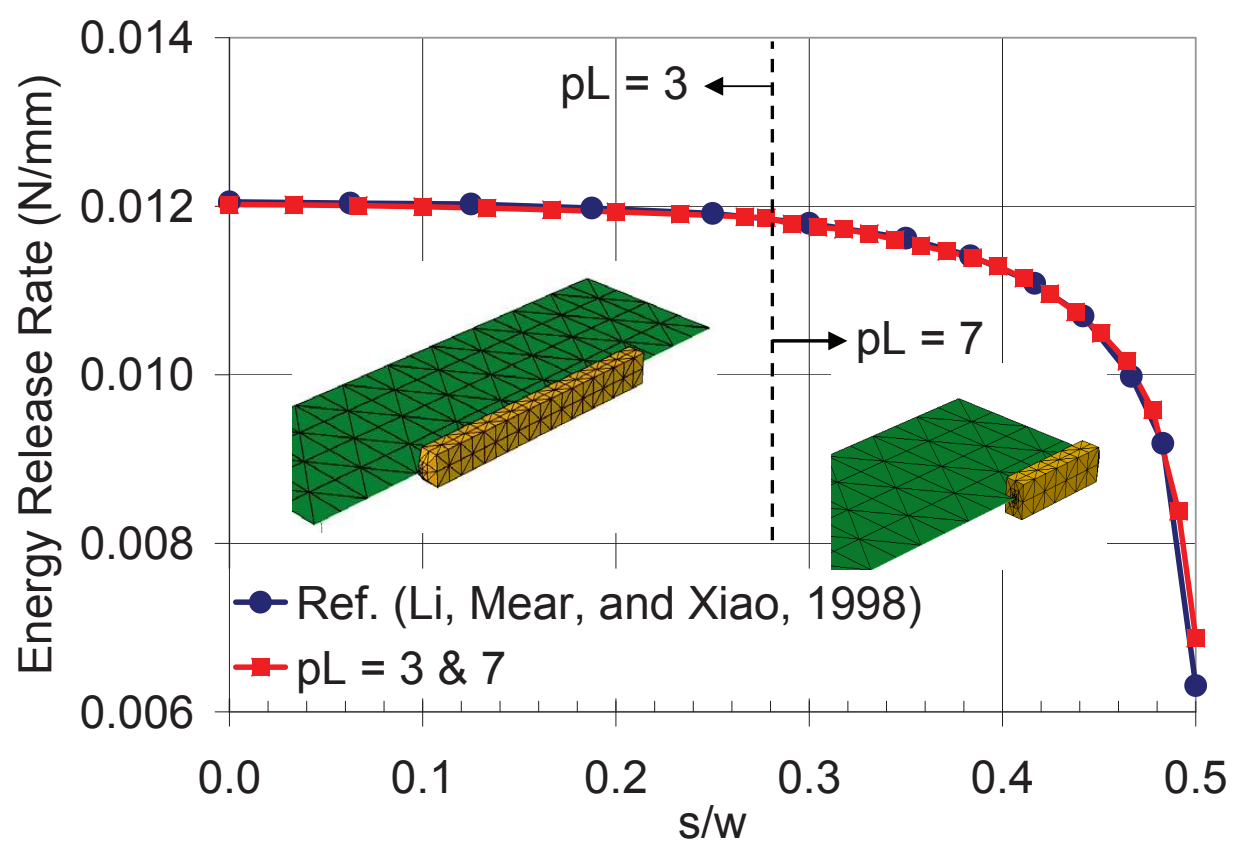

Figure 10: Comparison of high-order J-integral computed for the edge crack problem partitioning crack front to edge and center extraction domains ( $p L$ : polynomial order of the Legendre polynomials). 
patterns repeats itself for the three crack front refinement levels verifying the reliability of the formulation and implementation.

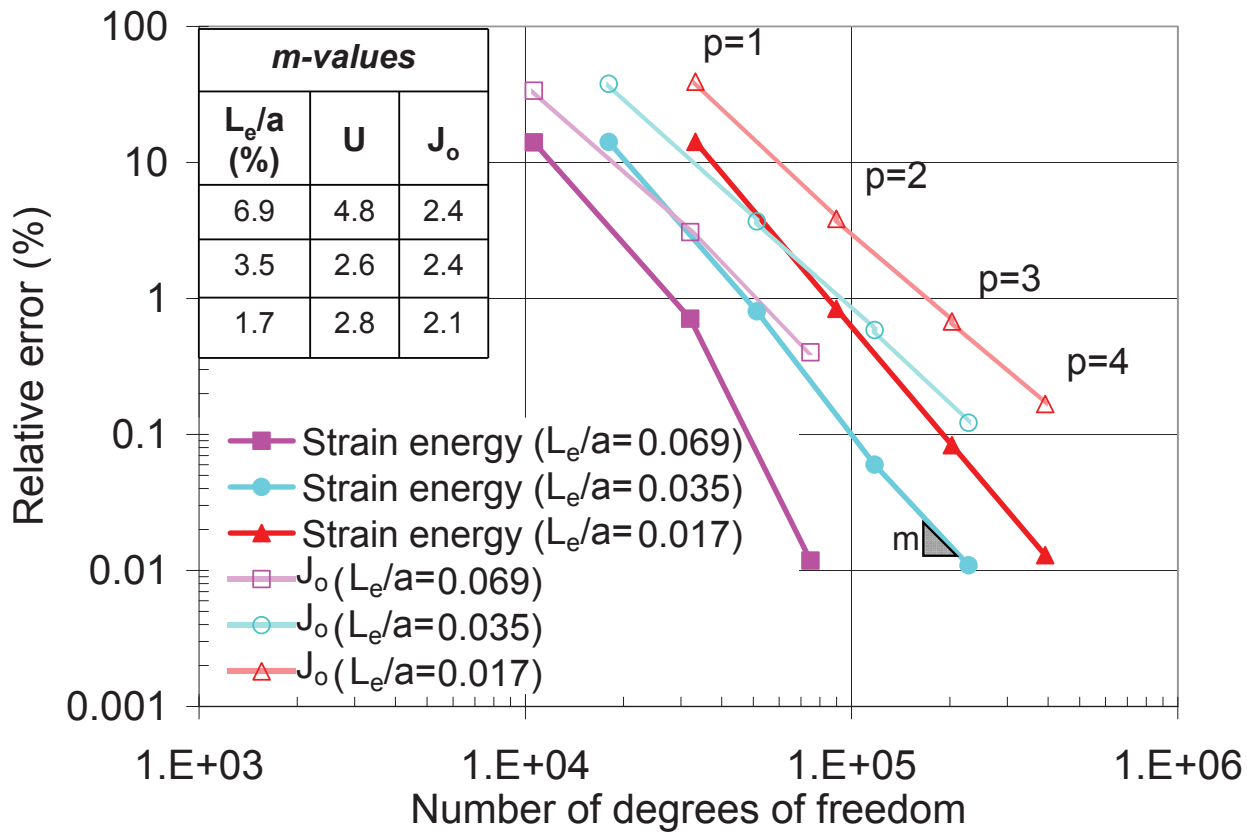

Figure 11: Convergence analysis of strain energy and energy release at three different crack front refinement levels ( $L_{e} / a=0.069, L_{e} / a=0.035$, and $\left.L_{e} / a=0.017\right)$ for the edge crack example (U: strain energy).

\subsection{Center Slanted Crack}

The second example consists of a slanted crack inserted in a finite size plate. The objective of this example is to illustrate the capabilities of the GFEM with the proposed domain integral implementation in solving a mixed mode fracture problem. Figure 12 shows the problem geometry and crack surface used in this problem. The bar is subjected to uniaxial remote tensile tractions. Young's modulus and Poisson's ratio are taken as 1000 and 0.33 , respectively. This problem was also solved by Cisilino and Ortiz Cisilino and Ortiz [2005] using a Boundary Element Method.

Locally refined meshes are used for the solution of the problem. Figure 13 shows a comparison between the results obtained from the standard domain integral and the reference values Cisilino and Ortiz [2005]. Crack front elements are locally refined to the level of $L_{e} / a$ ratio around 0.014 .

The results from the high-order domain integral formulation are also compared to the reference results found in the literature. Similar to the edge crack example, the order of Legendre polynomials are increased from 1 to 7 to compute the $J$-integral along the slanted crack fronts. The entire crack front domain is used as extraction domain to compute the $J$-integral. The results are shown in Figure 14. The approximation of $J$-integral is in good agreement with the reference values. The best results are obtained when the order of Legendre polynomials is chosen as 7.

Similar to the edge crack example, the crack front domain is also partitioned in edge and center extraction domains to improve the solution at the edges. Figure 15 illustrates these results. The order of Legendre polynomials is chosen compatible with the order of approximation at the center domain. Legendre polyno- 


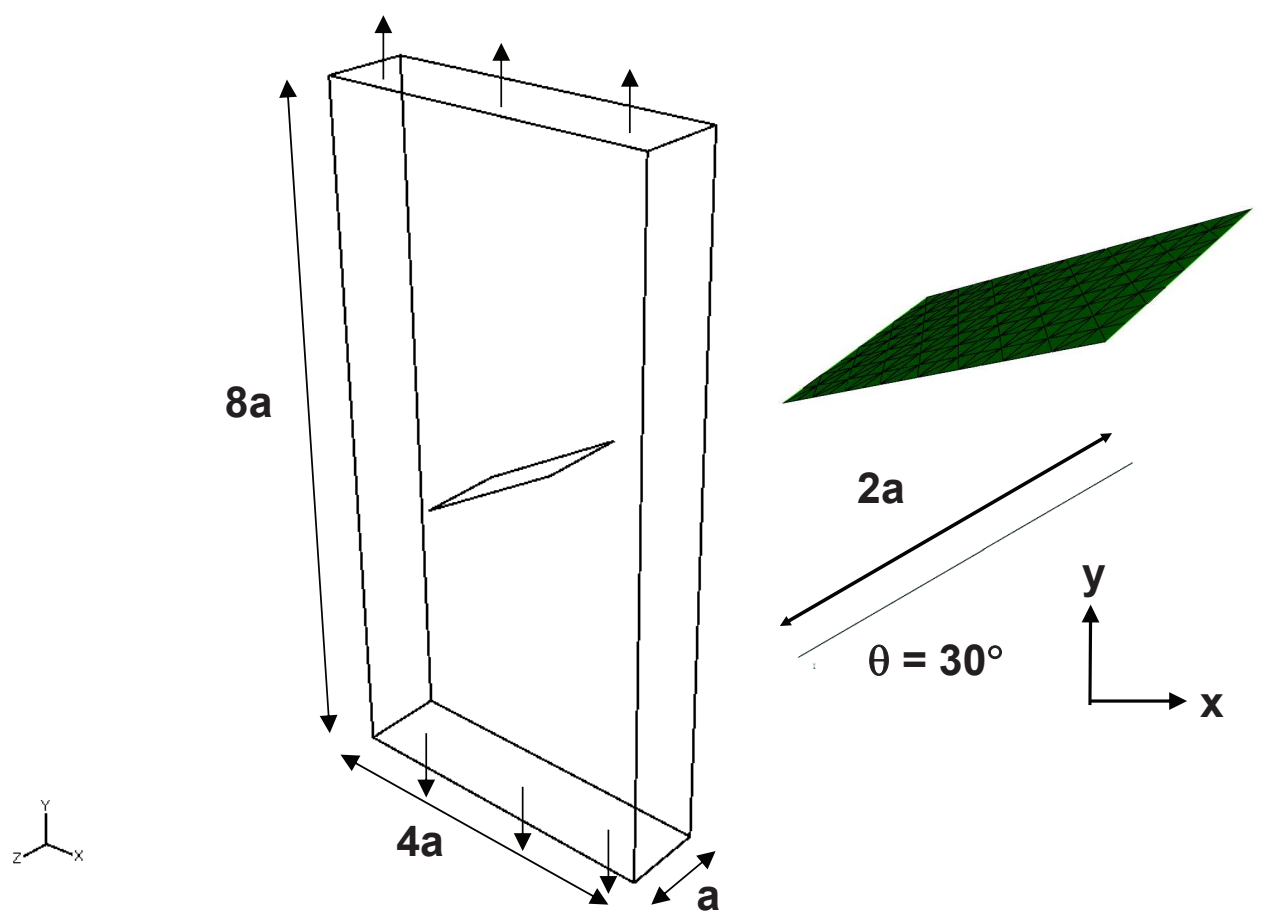

Figure 12: Center slanted crack in a plate subjected to uniaxial tensile tractions.

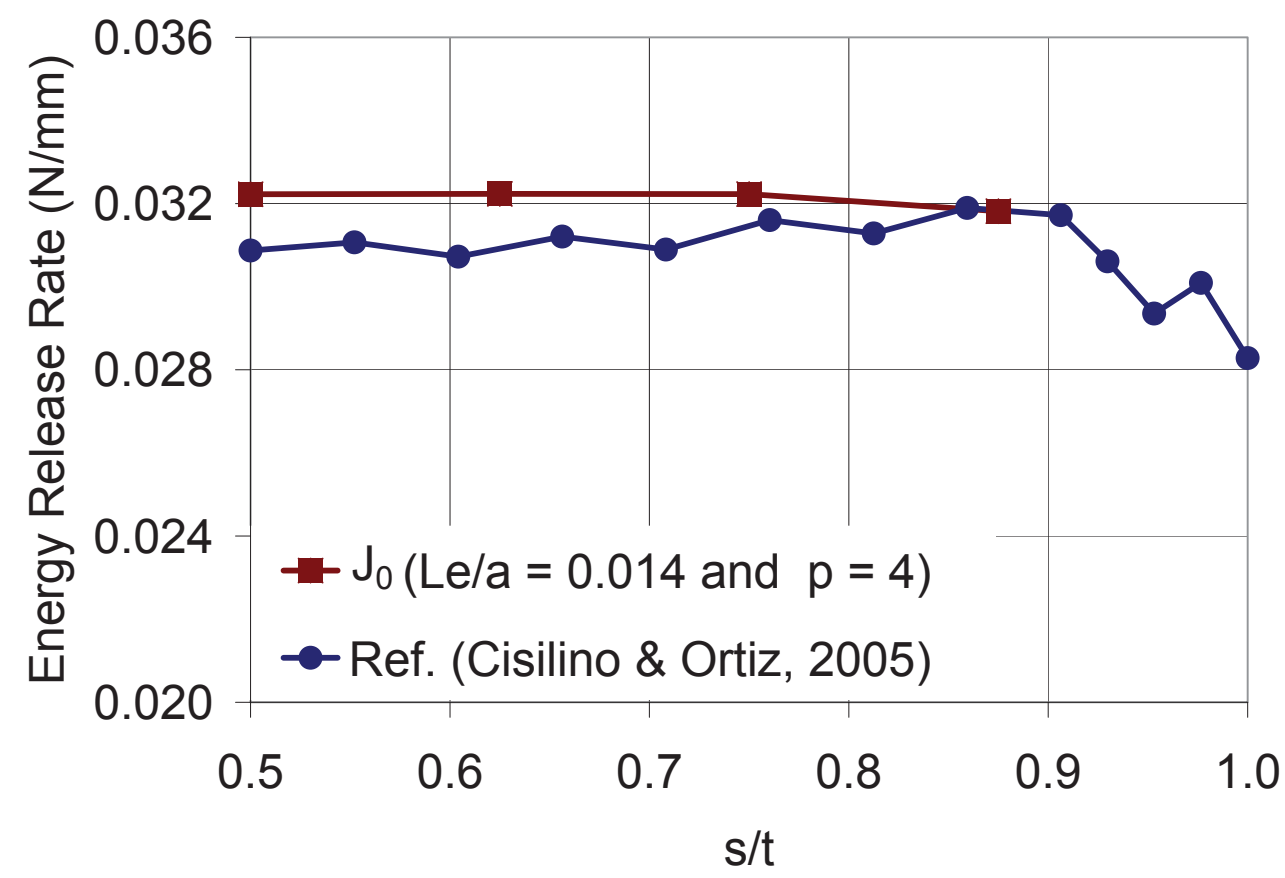

Figure 13: Comparison of the energy release rate computed along the crack front with the results from a reference solution for the center slanted crack example Cisilino and Ortiz [2005]. 


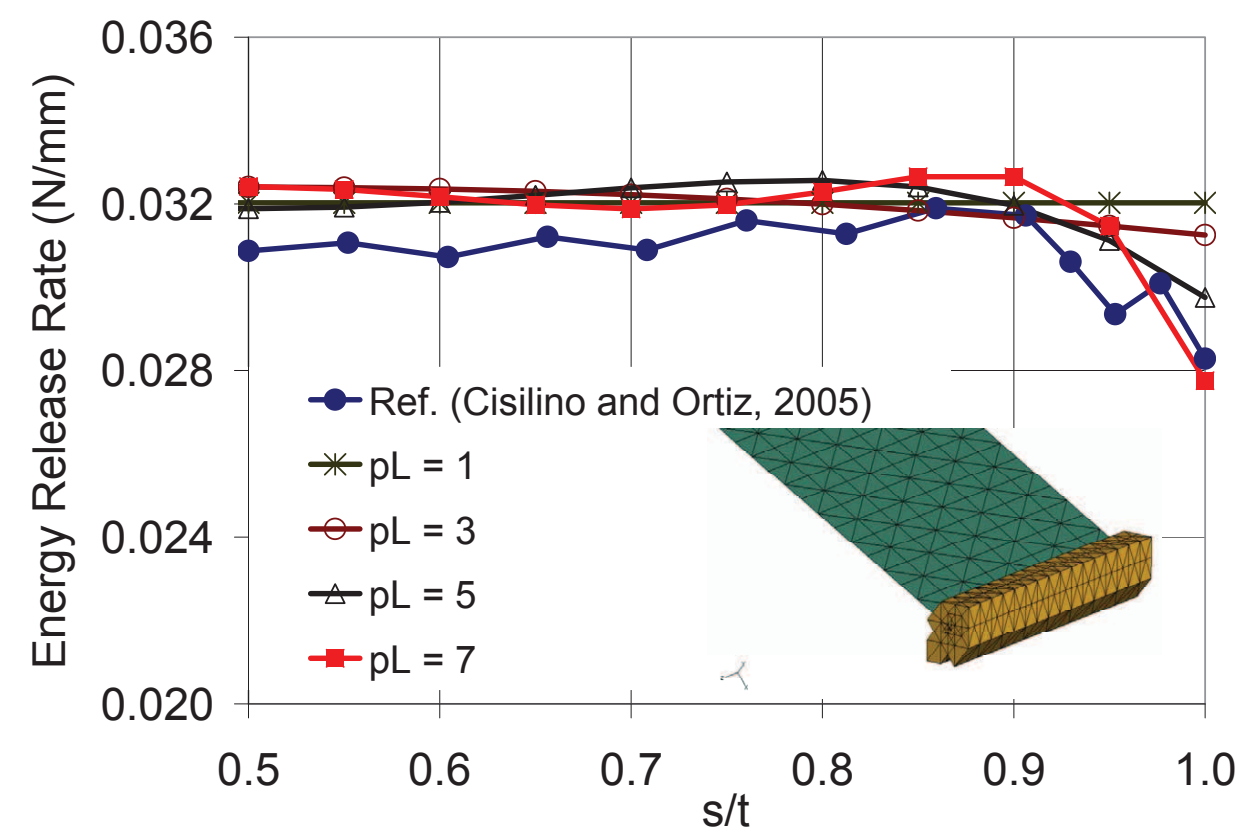

Figure 14: Comparison of high-order J-integral computed for the center slanted crack problem using whole crack front domain ( $p L$ : polynomial order of the Legendre polynomials).

mials of higher order are used at the crack front vertices to capture the gradient of $J$ that occur there. As shown in Figure 15, the solution is improved at the vertices similar to the results presented for the edge crack problem.

A convergence analysis is also performed. The order of the polynomial approximation is increased from $p=1$ to $p=3$ for various crack front refinement levels. Crack front elements are locally refined to the levels of $L_{e} / a$ ratios around $0.057,0.029$, and 0.014 , respectively. The value of the energy release rate computed at the middle of the crack front $(s / t=0.5)$ is used in the convergence analysis. Reference values for strain energy and energy release rate are obtained using a similar approach described in the edge crack example. The computed reference value of strain energy and energy release rate are $U_{\text {ref }}=0.032271775$ and $J_{\text {ref }}(s / t=0.5)=18.71918381$. Figure 16 shows the results of this convergence analysis. The slope of the curves are also illustrated as an insert to Figure 16.

\subsection{Circular Crack in a Finite Size Domain}

In this section, a circular crack of radius $a$ located at the center of a finite domain is considered. The problem geometry and the circular crack are illustrated in Figure 17. The objective of this problem is to test the robustness of the domain integral implementation for a curved crack front. The standard domain integral formulation is used for this example. A curved crack front poses a challenge in building extraction domains which can result in greatly unstructured and irregular meshes. This problem was also solved by $\mathrm{Li}$ et al. Li et al. [1998]. The crack is located at the center of a cube whose width is $2 w$. Crack size proportionality is taken as $a / w=0.5$. The domain is subjected to an axial uniform tension, $\sigma$. Young's modulus and Poisson's ratio are taken as 1 and 0.3 , respectively.

The problem is analyzed using the GFEM and the values of energy release rate are computed along the 


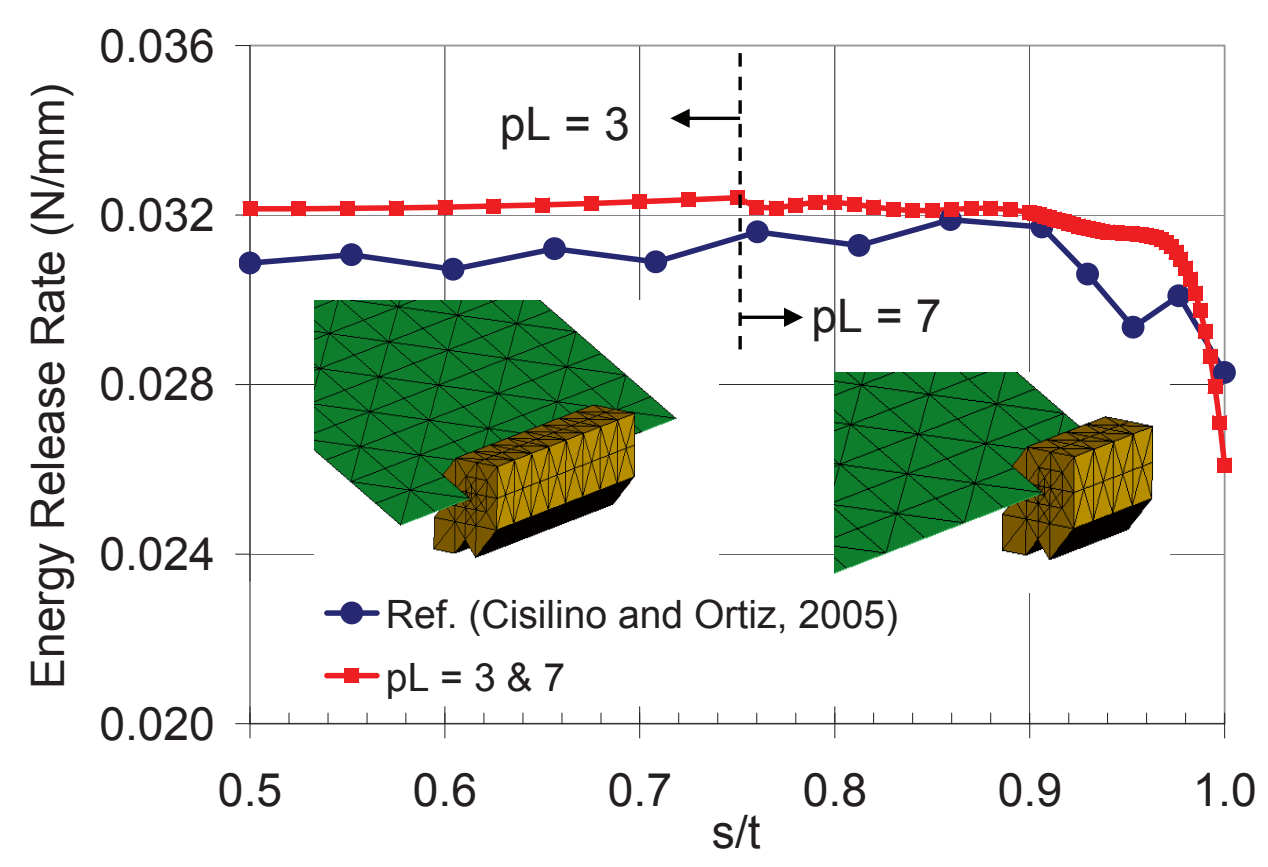

Figure 15: Comparison of high-order J-integral computed for the center slanted crack problem by partitioning the crack front to edge and center extraction domains ( $p L$ : polynomial order of the Legendre polynomials).

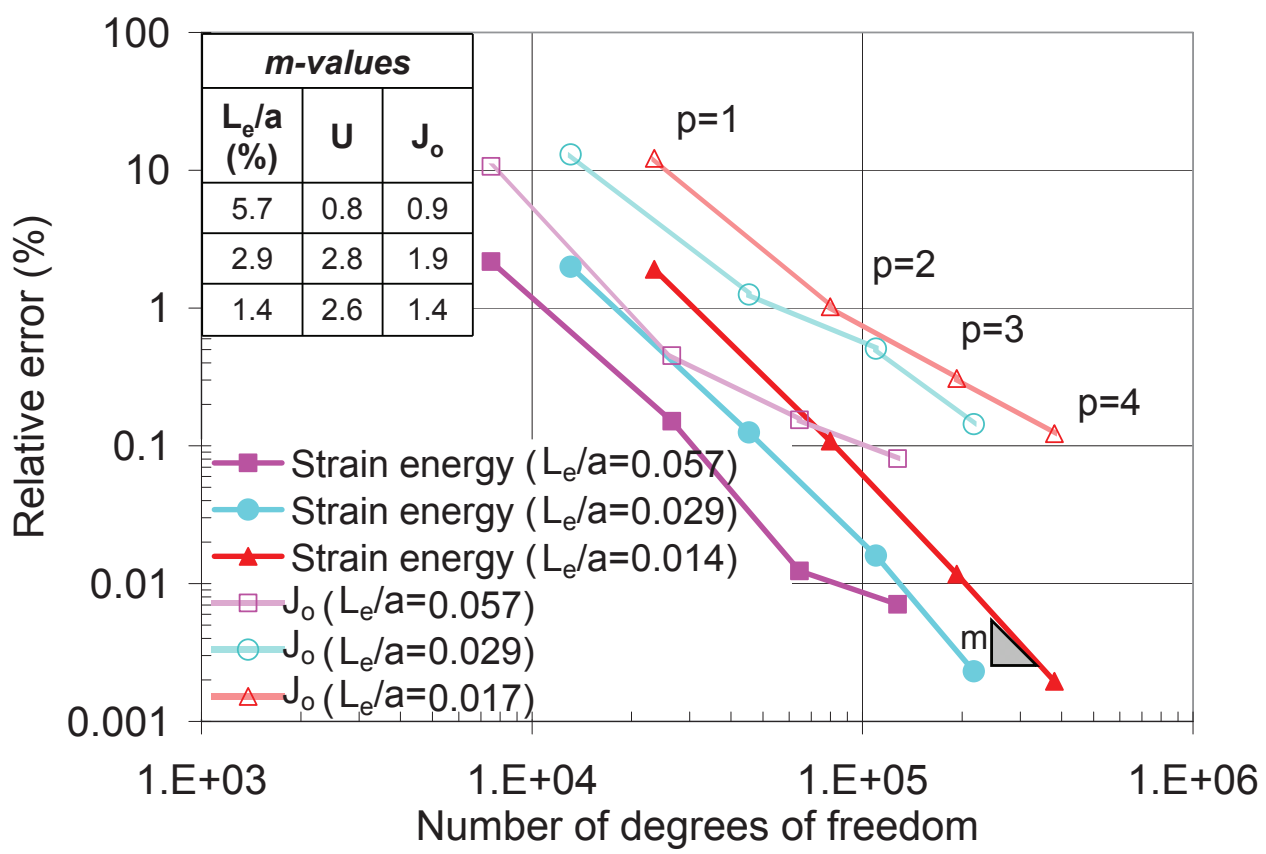

Figure 16: Convergence analysis of strain energy and energy release at three different crack front refinement levels ( $L_{e} / a=0.057, L_{e} / a=0.029$, and $\left.L_{e} / a=0.014\right)$ for the center slanted crack example ( $U$ : strain energy). 


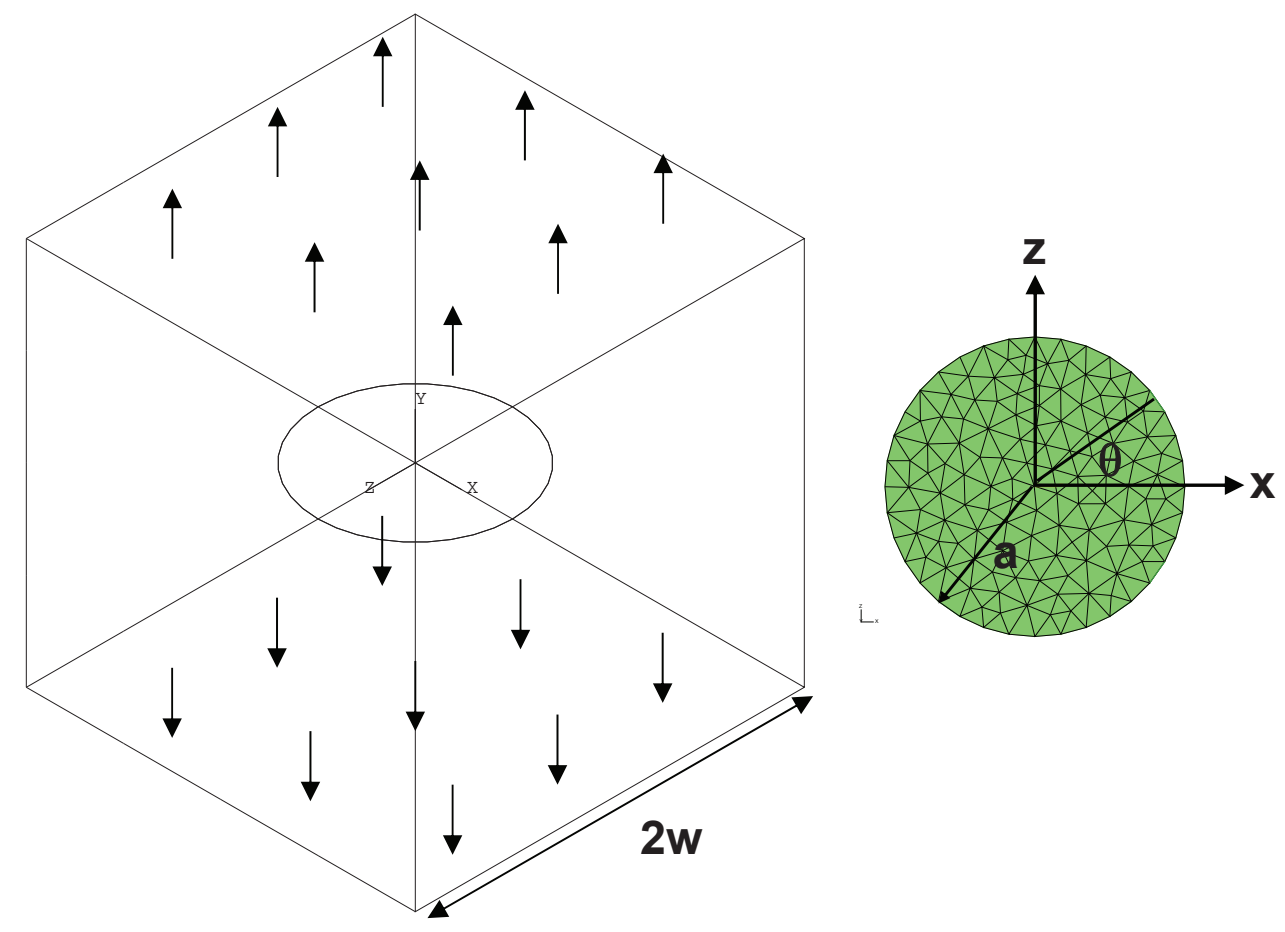

Figure 17: Circular crack in a cube subjected to uniform tensile tractions.

curved crack front at each vertex. Similar to the procedure followed in the previous examples, crack front elements are locally refined to the levels of ratios $L_{e} / a$ around $0.0884,0.0442$, and 0.0221 . The polynomial order of approximation varies from $p=1$ to $p=3$. The reference solution for stress intensity factor is given by Li et al. [1998] the following:

$$
K_{I}^{\mathrm{ref}}=\frac{2.213}{\pi} \sqrt{\pi a} \sigma
$$

A reference value for the energy release rate is then computed using

$$
J_{\text {ref }}=\frac{1+\kappa}{8 \mu}\left(K_{\mathrm{I}}^{\mathrm{ref}}\right)^{2}
$$

where $\kappa$ is the Kolosov constant and $\mu$ is Lame's constant.

The results obtained from the GFEM solution at the highest refinement level $L_{e} / a=0.0221$ and polynomial enrichment order $p=3$ are normalized by this reference solution to obtain $J / J_{\text {ref. }}$. Extraction domains are formed by adding three layers of elements to the crack front. The results are illustrated in Figure 18. Selected extraction domains used in the computation of energy release rate are also shown in this figure. It can be observed that the extraction domains can sometimes take very unstructured and irregular shapes. In spite of that, the results are in good agreement with the reference solution, demonstrating the robustness of the methodology used.

The convergence of the energy release rate is also compared to the strain energy convergence. The strain energy results and the energy release rate values are obtained from three refinement levels and the polynomial order mentioned earlier. The results are shown in Figure 19 with respect to the number of 

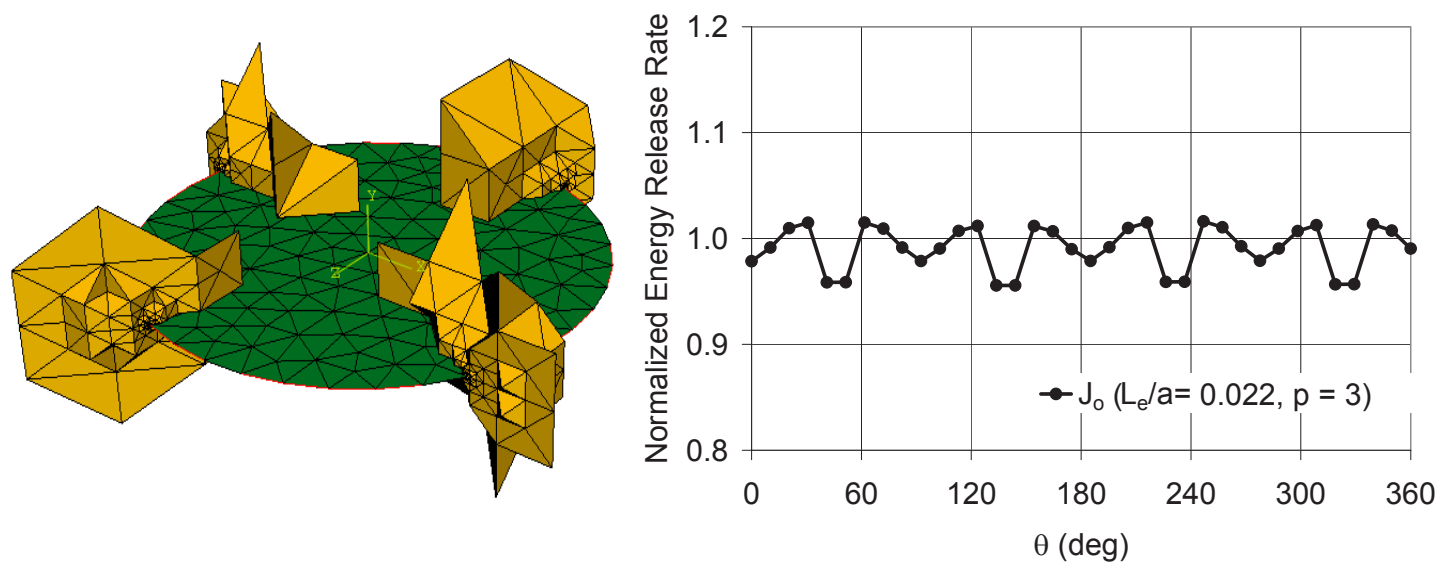

Figure 18: Normalized energy release rate along the crack front for the circular crack example.

degrees of freedom.

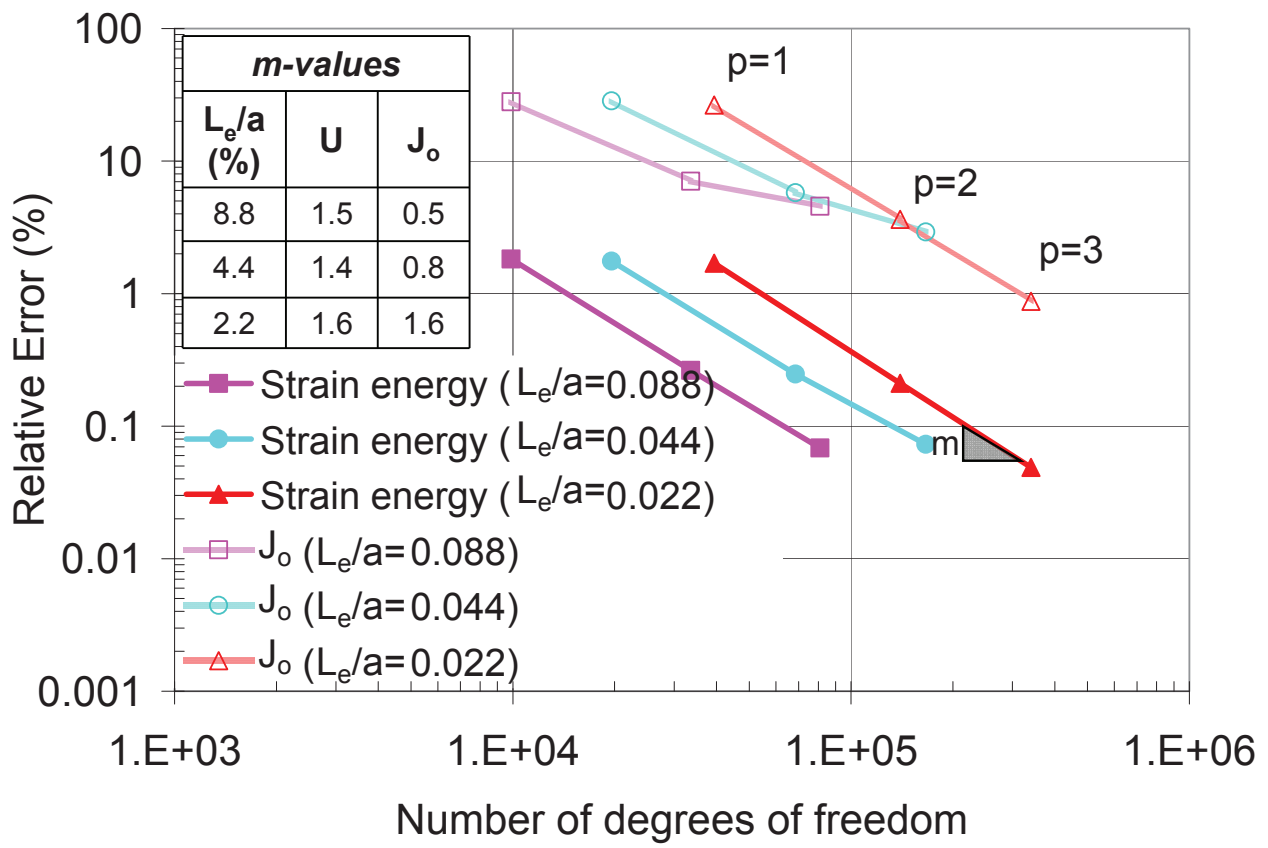

Figure 19: Convergence analysis of strain energy and energy release at three different crack front refinement levels $\left(L_{e} / a=0.088, L_{e} / a=0.044\right.$, and $\left.L_{e} / a=0.022\right)$ for the circular crack example ( $U$ : strain energy).

\subsection{A Non-Planar Crack Example}

The final example consists of a non-planar crack inserted in a finite size plate. The objective of this example is to illustrate the capabilities of the GFEM with the proposed domain integral implementation in 
solving a mixed mode fracture problem with non-planar cracks. As discussed in Section 2.2, our current implementation neglects the integrals over cracks surfaces $A_{5}$ and $A_{6}$. These integrals are non-zero in the case of non-planar crack surfaces. However, if the crack surface curvature is moderate within the extraction domain, the contributions from these surface integrals will be small. We test this conjecture in this example. The extraction domains adopted in the computations are shown as inserts in Figures 21 and 22.

Figure 20 shows the problem geometry and crack surface used in this problem. The non-planar crack has a circular arc shape with radius, $R$, and angle $80^{\circ}$. The plate is subjected to uniaxial remote tensile tractions. Young's modulus and Poisson's ratio are taken as 1000 and 0.33, respectively. This problem was also solved by Cisilino and Ortiz Cisilino and Ortiz [2005] using a Boundary Element Method. Similar to

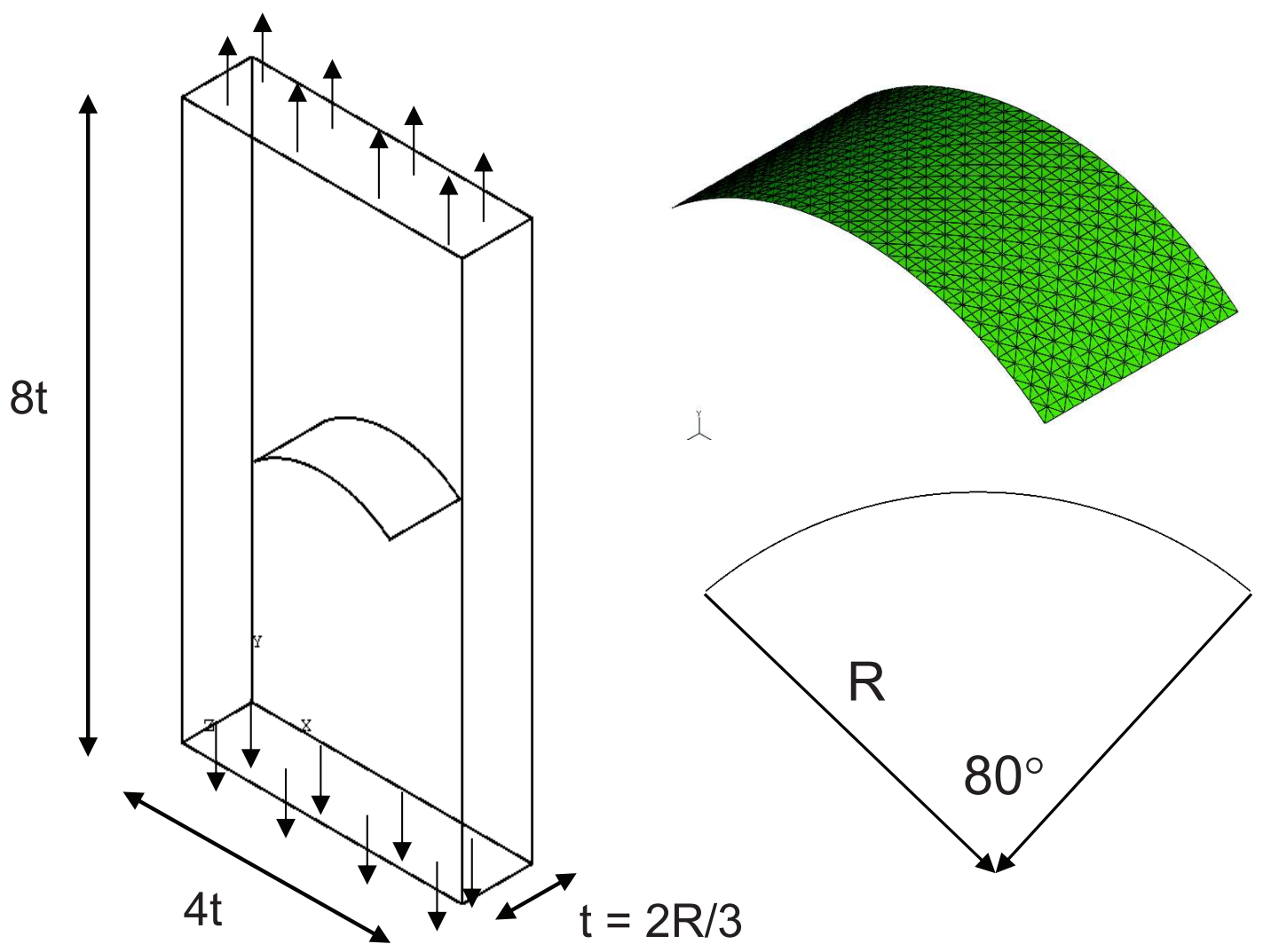

Figure 20: A non-planar circular arc crack in a plate subjected to uniform tensile tractions.

the previous examples, this one is solved using the high-order domain integral formulation. The results are obtained from the GFEM solution at refinement level $L_{e} / a=0.0135$ and polynomial enrichment order $p=3$. Figure 21 illustrates the $J$-integral computed using the whole crack front as extraction domain. It shows how the extracted $J$-integral evolves with increasing order of Legendre polynomials. However, as in previous examples, the $J$-integral cannot be captured well near the crack front edges where it exhibits a strong gradient. Therefore, partitioning the extraction domain is also required in this example to capture edge behavior. Figure 22 illustrates the results when the extraction domain is split into three subdomains; at the two edges and at the center of the crack. High gradients at the edges can now be captured. The order of Legendre polynomials at the edges is taken as 7 in order to capture the boundary-layer behavior at those regions. On the other hand, an accurate approximation of the $J$-integral at the center of the crack front can 
be achieved with quadratic polynomials.

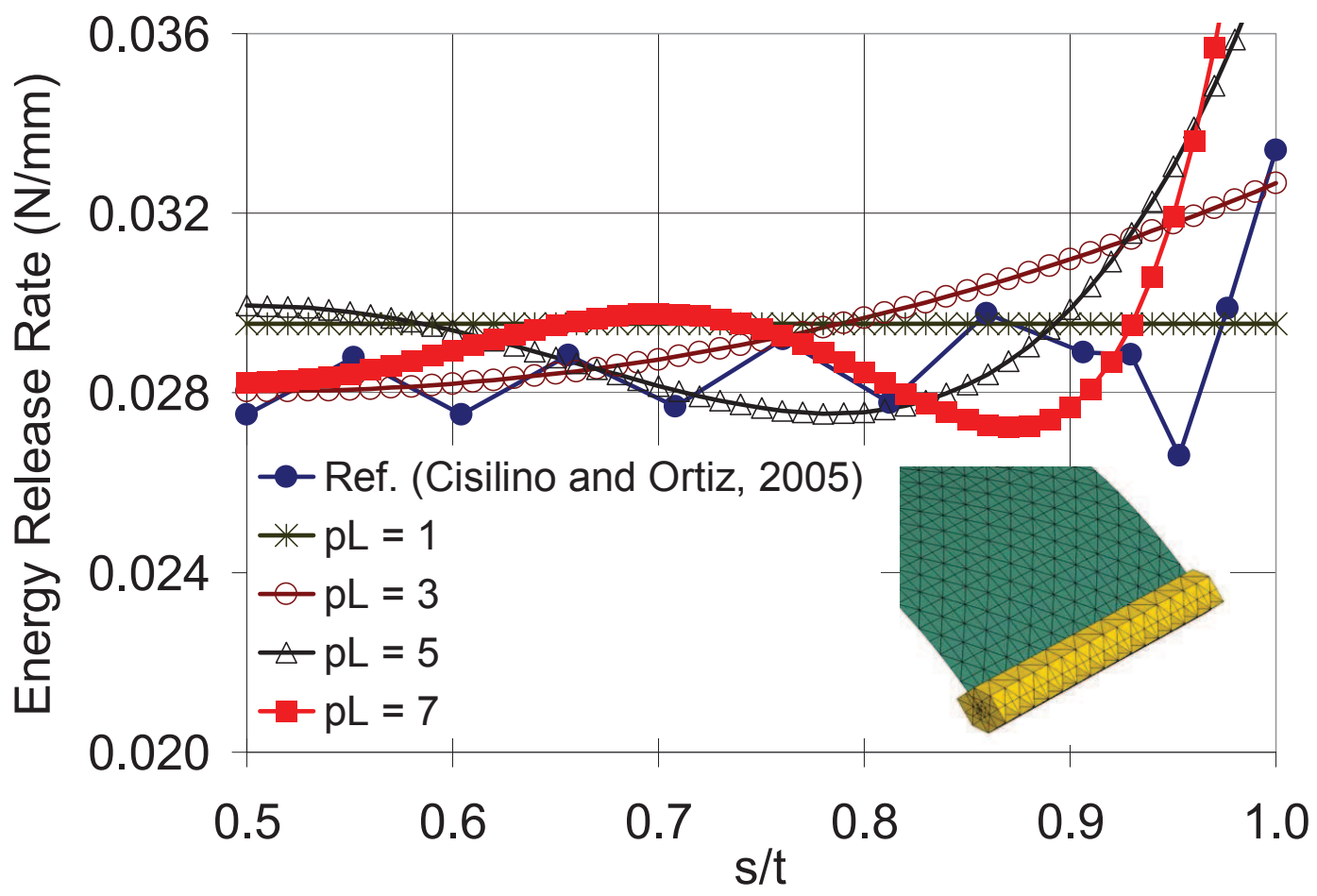

Figure 21: A comparison of the J-integral computed using high-order domain integral using varying orders of Legendre polynomials and the whole crack front as extraction domain.

\section{Conclusions}

A high-order domain integral method is presented. The domain integral method is used as a post processing technique to compute energy release rates along curved and straight 3-D crack fronts. The proposed implementation is compatible with the G/XFEM type of partition of unity based methods. A high-order domain integral method is formulated based on an approximation of the $J$-integral using Legendre polynomials. This technique yields the $J$-integral as a smoothly varying function along the crack front, which can be advantageous during propagation of crack fronts in 3-D fracture problems. A smooth function representing the stress intensity factors or energy release rate along a 3-D crack front can facilitate smooth propagation of the crack front segments. It is shown that the high-order domain integral reduces to the standard domain integral method when the order of the Legendre polynomial is taken as zero.

Special attention is given to the construction of extraction domains. The extraction domains consist of existing computational elements in which solution variables are already accessible. This implementation approach, using union of elements for the extraction domain, is particularly advantageous and yet challenging for partition of unity based methods such as GFEM/XFEM since the crack is independent of the problem discretization. The proposed implementation uses an element-by-element integration scheme in which discontinuities of the integrand across computational element boundaries and the crack surface are fully accounted for. 


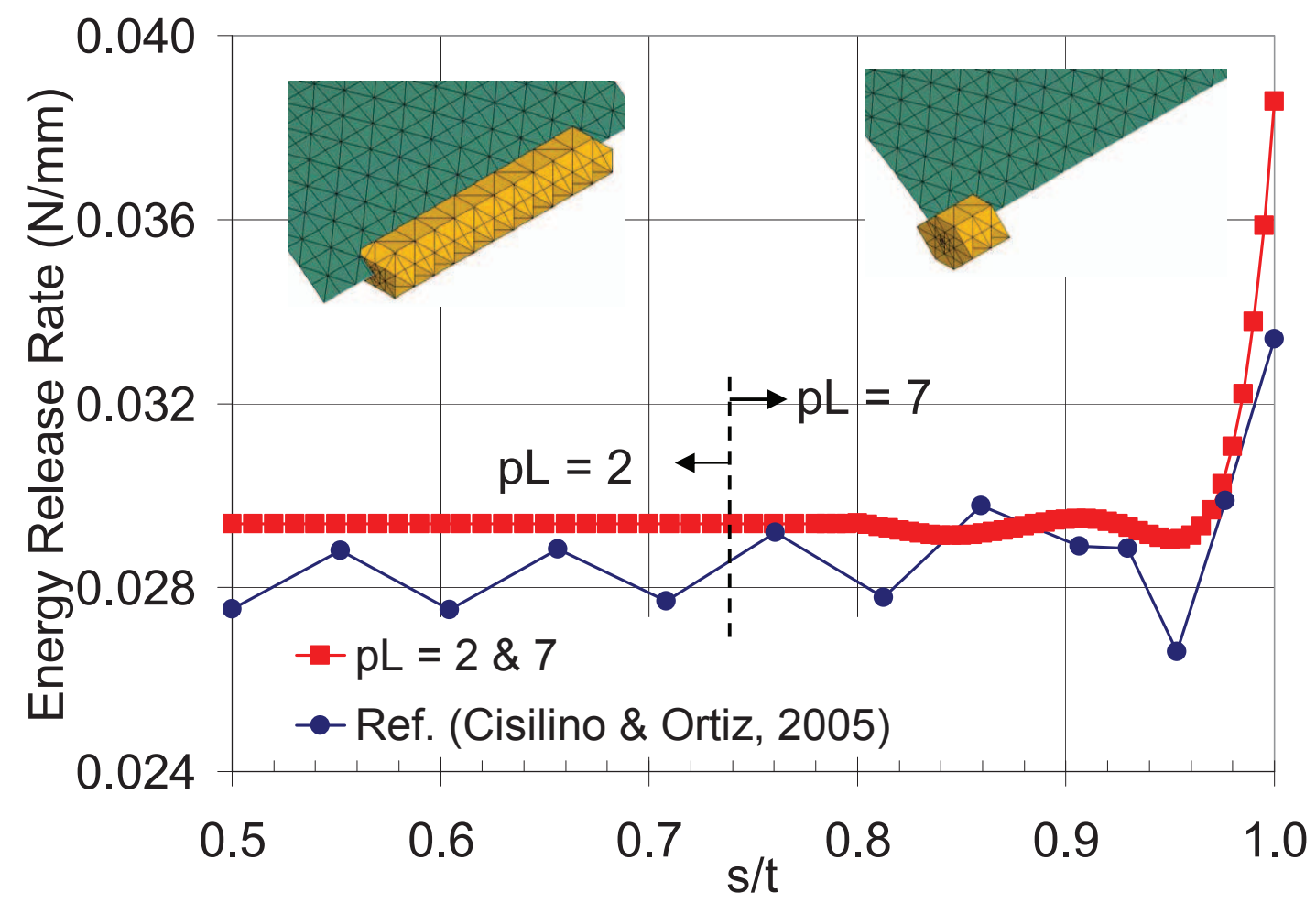

Figure 22: A comparison of the J-integral computed using high-order domain integral using varying orders of Legendre polynomials and partitioned crack front as extraction domains. 
The example problems are solved following the standard and the high-order domain integral formulation. Several benchmark problems are analyzed in order to examine the robustness, accuracy, and convergence of the proposed implementation. A p-convergence analysis is presented for problems with straight and curved cracks. In order to test the accuracy, results are compared to those obtained using other numerical techniques available in the literature. It is also shown that the proposed implementation possesses the property of domain independence.

Partitioning crack front extraction domains into edge and center domains is shown to be useful to capture the strong gradient of the $J$-integral near crack front vertices. The number of extraction domains along a crack front is, from the proposed formulation point of view, arbitrary. The numerical experiments presented in Section 4 show, however, that if a single extraction domain is used, very high order of approximation for the $J$-integral must be used. Thus, it is more computationally efficient to break the crack front in a few extraction sub-domains in the case of surface breaking cracks since the $J$-integral has, in general, a strong gradient near the breaking points while being smooth away from them. The selection of the size of these extractions domains is flexible and a quasi uniform sub-division of the crack front can be used as demonstrated in the numerical examples presented in Section 4.

The $J$-integral, as presented in this paper, can not extract stress intensity factors from a mixed mode problem. Extensions of the current formulation aimed at overcoming this limitation are under investigation by our research group and will be reported elsewhere.

\section{Acknowledgments}

The authors gratefully acknowledge the support provided by the NEXTRANS Center Project No. USDOTPU-4108-21574, which was conducted in cooperation with the Illinois Center for Transportation, Illinois Department of Transportation, and U. S. Department of Transportation, Federal Highway Administration. We also extend our thanks to Dr. Matthew C. Walters for his helpful suggestions. The contents of the paper reflect only the view of the authors, who are responsible for the facts and the accuracy of the data presented herein. This paper does not constitute a standard, specification, or regulation.

\section{References}

B. Andersson, U. Falk, I. Babuška, and T. Von Petersdoff. Reliable stress and fracture mechanics analysis of complex components using a hp version of FEM. International journal for numerical methods in engineering, 38(13):2135-2163, 1995. 2, 4

I. Babuška, G. Caloz, and J.E. Osborn. Special finite element methods for a class of second order elliptic problems with rough coefficients. SIAM Journal on Numerical Analysis, 31(4):945-981, 1994. 2, 8, 9

T. Belytschko, R. Gracie, and G. Ventura. A review of extended/generalized finite element methods for material modeling. Modelling and Simulations in Materials Science and Engineering, 17:24pp, 2009. http://dx.doi.org/10.1088/0965-0393/17/4/043001. 2

J. Cervenka and V. E. Saouma. Numerical evaluation of 3-D SIF for arbitrary finite element meshes. Engineering Fracture Mechanics, 57(5):541-563, 1997. 2 
A. P. Cisilino and J. Ortiz. Boundary element analysis of three-dimensional mixed-mode cracks via the interaction integral. Computer Methods in Applied Mechanics and Engineering, 194(9-11):935-956, 2005. 20, 21, 26

C.A. Duarte, I. Babuška, and J.T. Oden. Generalized finite element methods for three dimensional structural mechanics problems. Computers and Structures, 77:215-232, 2000. 2, 9

C.A. Duarte, O.N. Hamzeh, T.J. Liszka, and W.W. Tworzydlo. A generalized finite element method for the simulation of three-dimensional dynamic crack propagation. Computer Methods in Applied Mechanics and Engineering, 190(15-17):2227-2262, 2001. doi: 10.1016/S0045-7825(00)00233-4. 2, 9

C.A.M. Duarte and J.T. Oden. Hp clouds - An $h p$ meshless method. Numerical Methods for Partial Differential Equations, 12:673-705, 1996a. 2, 8, 9

C.A.M. Duarte and J.T. Oden. An $h p$ adaptive method using clouds. Computer Methods in Applied Mechanics and Engineering, 139:237-262, 1996b. 2, 8, 9

T.-P. Fries and T. Belytschko. The generalized/extended finite element method: An overview of the method and its applications. International Journal for Numerical Methods in Engineering, pages 253-304, 2010. 2

F. Z. Li, C. F. Shih, and A. Needleman. A comparison of methods for calculating energy release rates. Engineering Fracture Mechanics, 21(2):405-421, 1985. 1, 3

S. Li, M. E. Mear, and L. Xiao. Symmetric weak-form integral equation method for three-dimensional fracture analysis. Computer Methods in Applied Mechanics and Engineering, 151(3-4):435-459, 1998. $14,15,17,18,22,24$

J.M. Melenk and I. Babuška. The partition of unity finite element method: Basic theory and applications. Computer Methods in Applied Mechanics and Engineering, 139:289-314, 1996. 2, 8, 9

N. Moës, J. Dolbow, and T. Belytschko. A finite element method for crack growth without remeshing. International Journal for Numerical Methods in Engineering, 46:131-150, 1999. 11

N. Moës, A. Gravouil, and T. Belytschko. Non-planar 3D crack growth by the extended finite element and level sets-Part I: Mechanical model. International Journal for Numerical Methods in Engineering, 53: 2549-2568, 2002. 2

B. Moran and C. F. Shih. Crack tip and associated domain integrals from momentum and energy balance. Engineering Fracture Mechanics, 27(6):615-642, 1987. 1, 3, 4, 6, 8

G. P. Nikishkov and S. N. Atluri. Calculation of fracture mechanics parameters for an arbitrary threedimensional crack, by the 'equivalent domain integral' method. International Journal for Numerical Methods in Engineering, 24(9):1801-1821, 1987. 2, 12

J.P. Pereira and C.A. Duarte. Computation of stress intensity factors for pressurized cracks using the generalized finite element method and superconvergent extraction techniques. In P.R.M. Lyra, S.M.B.A. da Silva, F.S. Magnani, L.J. do N. Guimaraes, L.M. da Costa, and E. Parente Junior, editors, XXV Iberian Latin-American Congress on Computational Methods in Engineering, Recife, PE, Brazil, November 2004. 15 pages. ISBN Proceedings CD: 857409 869-8. 2 
J.P. Pereira and C.A. Duarte. Extraction of stress intensity factors from generalized finite element solutions. Engineering Analysis with Boundary Elements, 29:397-413, 2005. 2

J.P. Pereira and C.A. Duarte. The contour integral method for loaded cracks. Communications in Numerical Methods in Engineering, 22(5):421-432, 2006. doi: 10.1002/cnm.824. 2

J.P. Pereira, C.A. Duarte, D. Guoy, and X. Jiao. $\mathrm{Hp}$-Generalized FEM and crack surface representation for non-planar 3-D cracks. International Journal for Numerical Methods in Engineering, 77(5):601-633, 2009a. doi: 10.1002/nme.2419. 14, 15, 18

J.P. Pereira, C.A. Duarte, X. Jiao, and D. Guoy. Generalized finite element method enrichment functions for curved singularities in 3D fracture mechanics problems. Computational Mechanics, 44(1):73-92, 2009b. doi: 10.1007/s00466-008-0356-1. 15

J. R. Rice. A path independent integral and the approximate analysis of strain concentration by notches and cracks. Journal of applied mechanics, 35(2):379-386, 1968. 1

C. F. Shih, B. Moran, and T. Nakamura. Energy release rate along a three-dimensional crack front in a thermally stressed body. International Journal of Fracture, 30(2):79-102, 1986. 1, 3, 12

N. Sukumar, N. Moës, B. Moran, and T. Belytschko. Extended finite element method for three-dimensional crack modelling. International Journal for Numerical Methods in Engineering, 48:1549-1570, 2000. 2

B. Szabo and I. Babuška. Finite Element Analysis. John Wiley and Sons, New York, 1991. 2

B. A. Szabo and I. Babuška. Computation of the amplitude of stress singular terms for cracks and reentrant corners. In T.A. Cruse, editor, Fracture Mechanics: Nineteenth Symposium, ASTM STP 969, pages 101-124, Southwest Research Institute, San Antonio, TX, 1988. 2

B. A. Szabó and I. Babuška. Finite element analysis. Wiley-Interscience, 1991. 18 